Supporting Information

For

\title{
Rutile Promoted Synthesis of Sulfonylamidonitriles from Simple Aldehydes and Sulfonamides
}

\author{
Nicholas V. Costantini, ${ }^{2}$ Alex D. Bates, ${ }^{1}$ Graham J. Haun, ${ }^{1}$ Natasha M. Chang ${ }^{1}$ and Gustavo \\ Moura-Letts ${ }^{1^{*}}$ \\ ${ }^{1}$ Department of Chemistry and Biochemistry, College of Science and Mathematics \\ ${ }^{2}$ Department of Chemical Engineering, College of Engineering \\ Rowan University \\ 201 Mullica Hill Rd, Glassboro, NJ, 08028
}

\section{Supporting Information}
A. Materials and Methods
S2
B. Proposed reaction mechanism
S3
C. Synthesis of sulfonylamidonitriles from Table $\mathbf{2}$
S4
D. Synthesis of sulfenylamidonitriles from Table 3
S8
E. ${ }^{1} \mathrm{H}-\mathrm{NMR}$ and ${ }^{13} \mathrm{C}-\mathrm{MR}$ spectra
S11
F. References
S26 


\section{A. Materials AND Methods}

Reagents were obtained from Aldrich Chemical (www.sigma-aldrich.com), Acros Organics (www.us.vwr.com) or Alfa Aesar (www.us.vwr.com) and used without further purification. Solvents were obtained from EMD Miliphore DrySol (www.us.vwr.com) and degassed with $\mathrm{N}_{2}$. Solution phase reactions were performed in glass vials or round bottom flasks without inert atmosphere and magnetic stirring. Cold baths were generated as follows: $0{ }^{\circ} \mathrm{C}$, wet ice/water; $10{ }^{\circ} \mathrm{C}$, ice/acetone; $-20^{\circ} \mathrm{C}$, dry ice/isopropanol monitored with a thermometer; $-44{ }^{\circ} \mathrm{C}$, dry ice $/ \mathrm{CH}_{3} \mathrm{CN} ;-63^{\circ} \mathrm{C}$, dry ice/chloroform; $-78^{\circ} \mathrm{C}$, dry ice/acetone; $-100{ }^{\circ} \mathrm{C}$, dry ice/Et ${ }_{2} \mathrm{O}$.

TLC was performed on $0.25 \mathrm{~mm}$ E. Merck silica gel $60 \mathrm{~F} 254$ plates and visualized under UV light $(254 \mathrm{~nm})$ or by staining with potassium permanganate $\left(\mathrm{KMnO}_{4}\right)$, cerium ammonium molybdenate (CAM), phosphomolybdic acid (PMA), iodine $\left(I_{2}\right)$, or $p$-anisaldehyde. Silica flash chromatography was performed on E. Merck 230-400 mesh silica gel 60. Automated chromatography was performed on a ISOLERA Prime instrument with $10 \mathrm{~g}$. SNAP silica gel normal phase cartridges using a flow rate of $12.0 \mathrm{~mL} / \mathrm{min}$ and a gradient of $0-100 \%$ EtOAc in heptanes over $20 \mathrm{~min}$ with UV detection at $254 \mathrm{~nm}$. UPLC was carried out on a Agilent 1100 UPLC with a Phenomenex $5 \mathrm{~cm}$ ' $4.6 \mathrm{~mm}, 3 \mathrm{~mm}, 120 \AA$, C18 reverse phase column using a flow rate of $0.1 \mathrm{~mL} / \mathrm{min}$ and a gradient of $80-95 \% \mathrm{CH}_{3} \mathrm{CN}$ in $0.1 \%$ aq TFA over 5 min with UV detection at $254 \mathrm{~nm}$. Analytical LC-MS was carried out on a Agilent 1100 UPLC System with Ion Trap MS Detector with a Phenomenex $5 \mathrm{~cm}, 120 \AA$, C18 reverse phase column using a flow rate of $0.1 \mathrm{~mL} / \mathrm{min}$ and a gradient of $80-100 \% \mathrm{CH}_{3} \mathrm{CN}$ in $0.1 \%$ aq TFA over 5 min with UV detection at $254 \mathrm{~nm}$. NMR spectra were recorded on Varian Mercury II $400 \mathrm{MHz}$ Spectrometer at $24{ }^{\circ} \mathrm{C}$ in $\mathrm{CDCl}_{3}$ unless otherwise indicated. Chemical shifts are expressed in ppm relative to TMS $\left({ }^{1} \mathrm{H}\right.$, $0 \mathrm{ppm})$ or solvent signals: $\mathrm{CDCl}_{3}\left({ }^{1} \mathrm{H}, 7.23 \mathrm{ppm} ;{ }^{13} \mathrm{C}, 77.0 \mathrm{ppm}\right)$. Coupling constants are expressed in $\mathrm{Hz}$. 


\section{B. PRoposed REACTION MECHANISM:}

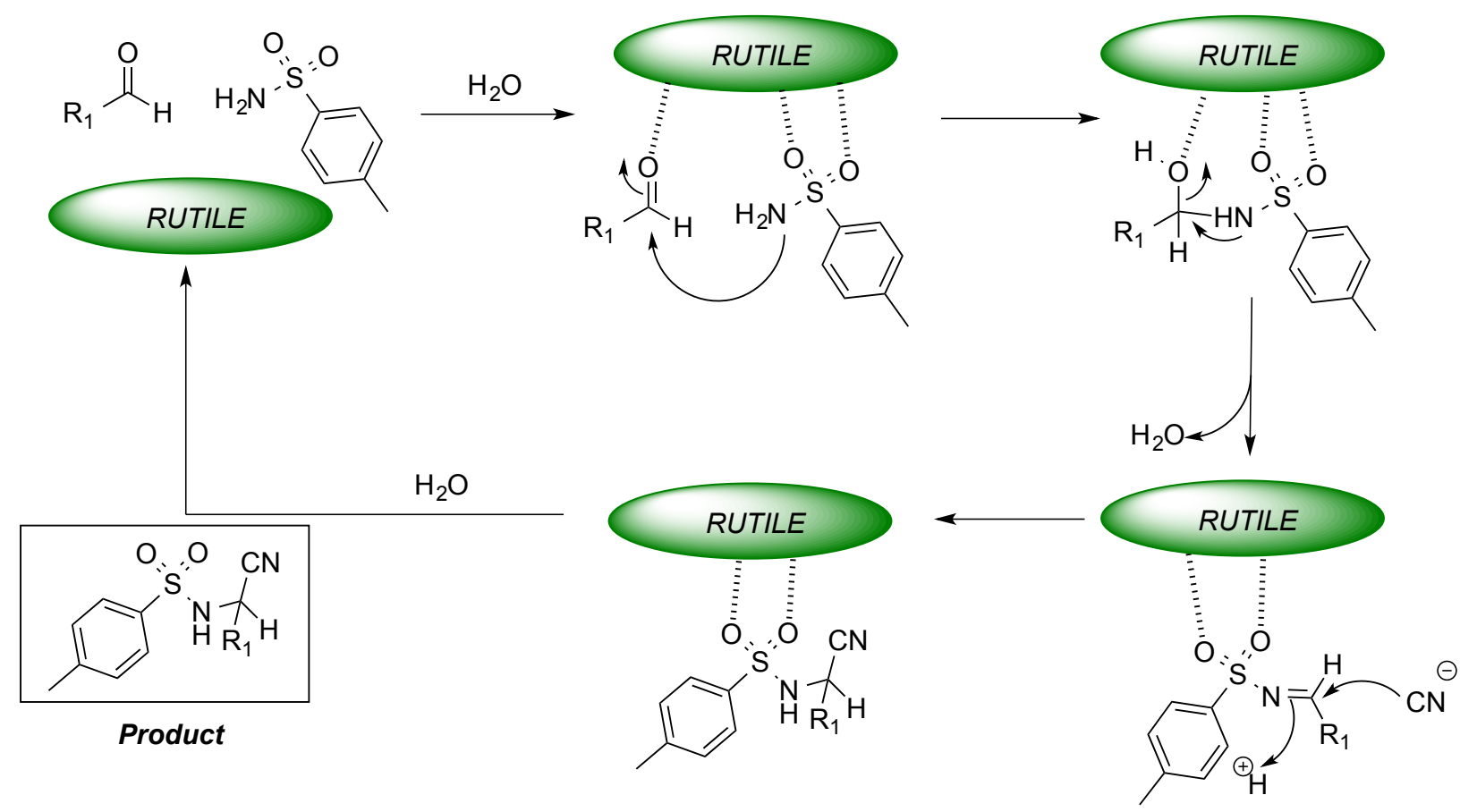

Figure S1. Proposed mechanism for rutile promoted synthesis of sulfonylamidonitriles. 


\section{SYNTHESIS OF SULFONYLAMIDONITRILES FROM TABLE 2:}

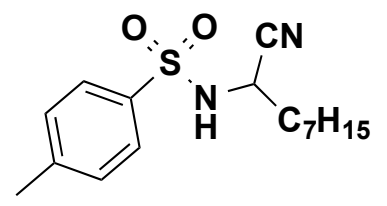

$\mathbf{N}$-(1-cyanooctyl)-4-methylbenzenesulfonamide (2a): Sulfonylamidonitrile 2a was obtained (27 mg, 88\%) as a pale-yellow oil. TLC: $R_{f} 0.64$ (2:1 heptanes/EtOAc). ${ }^{1} \mathrm{H}-\mathrm{NMR}\left(400 \mathrm{MHz}, \mathrm{CDCl}_{3}\right)$ : d $7.76(\mathrm{~d}, J=7.5 \mathrm{~Hz}, 2 \mathrm{H}), 7.28(\mathrm{~d}, J=7.5 \mathrm{~Hz}, 2 \mathrm{H}), 4.22(\mathrm{t}, J=7.1 \mathrm{~Hz}, 1 \mathrm{H}), 2.48(\mathrm{~s}, 3 \mathrm{H}), 1.75$ (ddd, J $=7.3,6.9,6.4 \mathrm{~Hz}, 2 \mathrm{H}), 1.48-1.44(\mathrm{~m}, 2 \mathrm{H}), 1.27-1.23(\mathrm{~m}, 8 \mathrm{H}), 0.82(\mathrm{t}, J=6.8 \mathrm{~Hz}, 3 \mathrm{H}) .{ }^{13} \mathrm{C}-\mathrm{NMR}$ $\left(100 \mathrm{MHz}_{1} \mathrm{CDCl}_{3}\right): \mathrm{d} 144.6,136.0,130.1,127.2,117.5,44.4,34.0,31.6,28.8,28.5,25.0,22.6$, 21.6, 14.0 ppm. ESI-MS m/z (rel int): (pos) 309.1 ([M+H $\left.]^{+}, 100\right)$; (neg) 307.1 ([M-H $\left.]^{-}, 100\right)$.

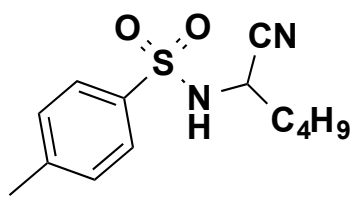

$\mathbf{N}$-(1-cyanohexyl)-4-methylbenzenesulfonamide (2b): Sulfonylamidonitrile $\mathbf{2 b}$ was obtained (25 mg, 91\%) as a colorless oil. TLC: $R_{f} 0.64$ (2:1 heptanes/EtOAc). ${ }^{1} \mathbf{H}-\mathbf{N M R}\left(400 \mathrm{MHz}, \mathrm{CDCl}_{3}\right)$ : d $7.78(\mathrm{~d}, J=7.3 \mathrm{~Hz}, 2 \mathrm{H}), 7.26(\mathrm{~d}, J=7.3 \mathrm{~Hz}, 2 \mathrm{H}), 6.02(\mathrm{bs}, 1 \mathrm{H}), 4.17(\mathrm{t}, J=6.8 \mathrm{~Hz}, 1 \mathrm{H}), 2.48(\mathrm{~s}$, $3 \mathrm{H}), 1.78(\mathrm{ddd}, J=7.5,7.0,6.4 \mathrm{~Hz}, 2 \mathrm{H}), 1.42-1.25(\mathrm{~m}, 4 \mathrm{H}), 0.75(\mathrm{t}, J=6.6 \mathrm{~Hz}, 3 \mathrm{H}) .{ }^{13} \mathrm{C}-\mathrm{NMR}$ $\left(100 \mathrm{MHz}, \mathrm{CDCl}_{3}\right): \mathrm{d}$ 144.3, 135.8, 129.8, 127.0, 117.5, 44.1, 33.1, 26.8, 21.5, 21.4, 13.4 ppm. ESI-MS m/z (rel int): (pos) $267.1\left([\mathrm{M}+\mathrm{H}]^{+}, 100\right)$; (neg) $265.1\left([\mathrm{M}-\mathrm{H}]^{-}, 100\right)$.

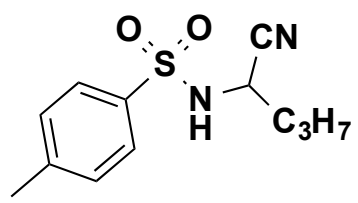

$\mathbf{N}$-(1-cyanobutyl)-4-methylbenzenesulfonamide (2c): Sulfonylamidonitrile 2c was obtained (22 mg, 90\%) as a colorless oil. ${ }^{1}$ TLC: $R_{f} 0.64$ (2:1 heptanes/EtOAc). ${ }^{1} \mathrm{H}-\mathrm{NMR}\left(400 \mathrm{MHz}, \mathrm{CDCl}_{3}\right)$ : d $7.81(\mathrm{~d}, J=7.2 \mathrm{~Hz}, 2 \mathrm{H}), 7.28(\mathrm{~d}, J=7.2 \mathrm{~Hz}, 2 \mathrm{H}), 5.78(\mathrm{bs}, 1 \mathrm{H}), 4.20(\mathrm{bs}, 1 \mathrm{H}), 2.48(\mathrm{~s}, 3 \mathrm{H}), 1.78$ $(\mathrm{dt}, J=7.3,6.9 \mathrm{~Hz}, 2 \mathrm{H}), 1.52-1.49(\mathrm{~m}, 2 \mathrm{H}), 0.98(\mathrm{t}, J=6.8 \mathrm{~Hz}, 3 \mathrm{H}) .{ }^{13} \mathrm{C}-\mathrm{NMR}\left(100 \mathrm{MHz}, \mathrm{CDCl}_{3}\right)$ : d 144.6, 135.9, 130.0, 127.2, 117.5, 44.1, 35.9, 21.6, 18.4, 13.0 ppm. ESI-MS m/z (rel int): (pos) $253.2\left([\mathrm{M}+\mathrm{H}]^{+}, 100\right)$; (neg) $251.2\left([\mathrm{M}-\mathrm{H}]^{-}, 100\right)$.<smiles>Cc1ccc(S(=O)(=O)NC(C#N)C(C)C)cc1</smiles>

$\mathbf{N}$-(1-cyano-2-methylpropyl)-4-methylbenzenesulfonamide (2d): Sulfonylamidonitrile 2d was obtained (25 mg, 99\%) as a pale-yellow oil. ${ }^{2}$ TLC: $R_{f} 0.64$ (2:1 heptanes/EtOAc). ${ }^{1} \mathbf{H}-\mathbf{N M R}$ (400 MHz, $\mathrm{CDCl}_{3}$ ): d $7.75(\mathrm{~d}, J=7.1 \mathrm{~Hz}, 2 \mathrm{H}$ ), $7.29(\mathrm{~d}, J=7.1 \mathrm{~Hz}, 2 \mathrm{H}), 5.58(\mathrm{~d}, J=5.7 \mathrm{~Hz}, 1 \mathrm{H}), 4.02$ 
$(\mathrm{d}, J=6.2 \mathrm{~Hz}, 1 \mathrm{H}), 2.48(\mathrm{~s}, 3 \mathrm{H}), 1.78(\mathrm{sd}, J=7.3,6.8 \mathrm{~Hz}, 1 \mathrm{H}), 1.03(\mathrm{~d}, J=7.3 \mathrm{~Hz}, 3 \mathrm{H}), 1.01(\mathrm{~d}, J=$ $7.3 \mathrm{~Hz}, 3 \mathrm{H}) .{ }^{13} \mathrm{C}-\mathrm{NMR}\left(100 \mathrm{MHz}, \mathrm{CDCl}_{3}\right)$ : d 144.3, 135.9, 129.9, 127.0, 116.6, 50.5, 32.2, 21.5, 18.3, 17.6 ppm. ESI-MS m/z (rel int): (pos) 253.2 ([M+H $\left.]^{+}, 100\right)$; (neg) $251.2\left([\mathrm{M}-\mathrm{H}]^{-}, 100\right)$.

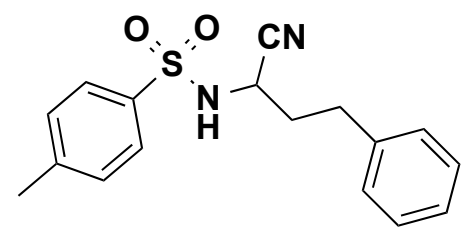

$\mathbf{N}$-(1-cyano-3-phenylpropyl)-4-methylbenzenesulfonamide (2e): Sulfonylamidonitrile $\mathbf{2 e}$ was obtained (29 mg, 92\%) as a pale-yellow oil. ${ }^{3}$ TLC: $R_{f} 0.68$ (2:1 heptanes/EtOAc). ${ }^{1} \mathrm{H}-\mathrm{NMR}$ $\left(400 \mathrm{MHz}, \mathrm{CDCl}_{3}\right): \mathrm{d} 7.76(\mathrm{~d}, J=7.3 \mathrm{~Hz}, 2 \mathrm{H}), 7.28-7.24(\mathrm{~m}, 5 \mathrm{H}), 7.09(\mathrm{~d}, J=7.3 \mathrm{~Hz}, 2 \mathrm{H}), 6.11$ (bs, $1 \mathrm{H}), 4.22(\mathrm{t}, J=7.1 \mathrm{~Hz}, 1 \mathrm{H}), 2.77(\mathrm{t}, J=6.6 \mathrm{~Hz}, 2 \mathrm{H}), 2.48(\mathrm{~s}, 3 \mathrm{H}), 2.06(\mathrm{tt}, J=7.1,6.6 \mathrm{~Hz}, 2 \mathrm{H})$. ${ }^{13}$ C-NMR $\left(100 \mathrm{MHz}_{\mathrm{CDCl}}\right)$ : d 144.3, 138.7, 135.6, 129.8, 128.5, 128.1, 127.0, 126.4, 117.3, 43.4, 34.9, 30.8, 21.3 ppm. ESI-MS m/z (rel int): (pos) 315.1 ([M+H $\left.]^{+}, 100\right)$; (neg) $313.1\left([\mathrm{M}-\mathrm{H}]^{-}, 100\right)$.<smiles>Cc1ccc(S(=O)(=O)NC(C#N)c2ccccc2)cc1</smiles>

$\mathbf{N}$-(cyano(phenyl)methyl)-4-methylbenzenesulfonamide (2f): Sulfonylamidonitrile $2 \mathrm{ff}$ was obtained ( $25 \mathrm{mg}, 89 \%$ ) as a colorless oil. ${ }^{4}$ TLC: $R_{f} 0.60$ (2:1 heptanes/EtOAc). ${ }^{1} \mathrm{H}-\mathrm{NMR}(400 \mathrm{MHz}$, $\left.\mathrm{CDCl}_{3}\right): \mathrm{d} 7.72(\mathrm{~d}, J=7.1 \mathrm{~Hz}, 2 \mathrm{H}), 7.25-7.22(\mathrm{~m}, 5 \mathrm{H}), 7.01(\mathrm{~d}, J=7.1 \mathrm{~Hz}, 2 \mathrm{H}), 6.28(\mathrm{bs}, 1 \mathrm{H}), 4.28(\mathrm{~s}$, $1 \mathrm{H}), 2.48(\mathrm{~s}, 3 \mathrm{H}) .{ }^{13} \mathrm{C}-\mathrm{NMR}\left(100 \mathrm{MHz}, \mathrm{CDCl}_{3}\right): \mathrm{d} 144.7,139.1,135.2,129.6,128.3,128.0,127.5$, 126.3, 116.0, 43.4, 21.3 ppm. ESI-MS m/z (rel int): (pos) 287.2 ([M+H] $\left.]^{+}, 100\right)$; (neg) 285.2 ([M$\left.\mathrm{H}]^{-}, 100\right)$.<smiles>Cc1ccc(C(C#N)NS(=O)(=O)c2ccc(C)cc2)cc1</smiles>

$\mathbf{N}$-(cyano(p-tolyl)methyl)-4-methylbenzenesulfonamide (2): Sulfonylamidonitrile 2 was obtained (29 mg, 97\%) as a pale-yellow oil. ${ }^{5}$ TLC: $R_{f} 0.62$ (2:1 heptanes/EtOAc). ${ }^{1} \mathbf{H}-\mathbf{N M R}$ $\left(400 \mathrm{MHz}, \mathrm{CDCl}_{3}\right)$ : d $7.76(\mathrm{~d}, J=7.1 \mathrm{~Hz}, 2 \mathrm{H}), 7.29$ (d, J= $\left.7.2 \mathrm{~Hz}, 2 \mathrm{H}\right), 7.25(\mathrm{~d}, J=7.2 \mathrm{~Hz}, 2 \mathrm{H}), 7.19$

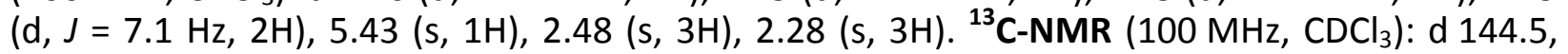
139.9, 136.0, 130.0, 129.6, 129.1, 127.3, 127.0, 116.5, 47.9, 21.6, $21.1 \mathrm{ppm}$. ESI-MS m/z (rel int): (pos) 301.1 ([M+H] $\left.]^{+}, 100\right)$; (neg) 299.1 ([M-H] $\left.]^{-}, 100\right)$. 
<smiles>COc1ccc(C(C#N)NS(=O)(=O)c2ccc(C)cc2)cc1</smiles>

$\mathrm{N}$-(cyano(4-methoxyphenyl)methyl)-4-methylbenzenesulfonamide $(\mathbf{2 g})$ : Purification by automated silica gel flash chromatography (10 g cartridge, $12 \mathrm{ml} / \mathrm{min}$. 20:1 heptanes/EtOAc to 1:4 heptanes/EtOAc over $12 \mathrm{~min}$ ) yielded sulfonylamidonitrile $\mathbf{2 g}(29 \mathrm{mg}, 91 \%)$ as a white solid. ${ }^{1}$ TLC: $R_{f} 0.42$ (2:1 heptanes/EtOAc). ${ }^{1} \mathrm{H}-\mathrm{NMR}\left(400 \mathrm{MHz}, \mathrm{CDCl}_{3}\right): \mathrm{d} 7.76(\mathrm{~d}, J=7.1 \mathrm{~Hz}, 2 \mathrm{H}), 7.34-$ $7.32(\mathrm{~m}, 4 \mathrm{H}), 7.28(\mathrm{~d}, J=7.1 \mathrm{~Hz}, 2 \mathrm{H}), 5.32(\mathrm{~s}, 1 \mathrm{H}), 3.76(\mathrm{~s}, 3 \mathrm{H}), 2.48(\mathrm{~s}, 3 \mathrm{H}) .{ }^{13} \mathrm{C}-\mathrm{NMR}(100 \mathrm{MHz}$, $\left.\mathrm{CDCl}_{3}\right)$ : d 160.4, 144.5, 135.9, 129.9, 128.5, 127.2, 124.0, 116.5, 114.5, 55.3, 47.6, 21.6 ppm. ESI-MS $m / z$ (rel int): (pos) $317.2\left([\mathrm{M}+\mathrm{H}]^{+}, 100\right)$; (neg) $315.2\left([\mathrm{M}-\mathrm{H}]^{-}, 100\right)$.<smiles>Cc1ccc(S(=O)(=O)NC(C#N)c2ccc3ccccc3c2)cc1</smiles>

$\mathbf{N}$-(cyano(naphthalen-2-yl)methyl)-4-methylbenzenesulfonamide (2h): Sulfonylamidonitrile $\mathbf{2 h}$ was obtained $(28 \mathrm{mg}, 85 \%)$ as a colorless oil. ${ }^{2}$ TLC: $R_{f} 0.55$ (2:1 heptanes/EtOAc). ${ }^{1} \mathrm{H}-\mathrm{NMR}$ (400 MHz, CDCl 3 ): d $7.99(\mathrm{~d}, J=7.1 \mathrm{~Hz}, 1 \mathrm{H}), 7.81(\mathrm{~d}, J=7.2 \mathrm{~Hz}, 2 \mathrm{H}), 7.76(\mathrm{~d}, J=7.2 \mathrm{~Hz}, 2 \mathrm{H}), 7.67$ $(\mathrm{d}, J=7.1 \mathrm{~Hz}, 1 \mathrm{H}), 7.54(\mathrm{t}, J=7.1 \mathrm{~Hz}, 2 \mathrm{H}), 7.29(\mathrm{t}, J=7.2 \mathrm{~Hz}, 1 \mathrm{H}), 7.23(\mathrm{~d}, J=7.2 \mathrm{~Hz}, 2 \mathrm{H}), 6.02$ (bs, 1H), 5.49 (bs, $1 \mathrm{H}), 2.46(\mathrm{~s}, 3 \mathrm{H}) .{ }^{13} \mathrm{C}-\mathrm{NMR}\left(100 \mathrm{MHz}, \mathrm{CDCl}_{3}\right): \mathrm{d}$ 144.6, 135.6, 133.9, 131.1, 129.9, 129.0, 127.8, 127.3, 126.9, 126.6, 126.5, 124.9, 122.3, 116.4, 46.3, 21.6 ppm. ESI-MS m/z (rel int): (pos) $337.2\left([\mathrm{M}+\mathrm{H}]^{+}, 100\right)$; (neg) $335.2\left(\left[\mathrm{M}-\mathrm{H}^{-}, 100\right)\right.$.<smiles>Cc1ccc(S(=O)(=O)NC(C#N)c2ccc3c(c2)OCO3)cc1</smiles>

$\mathrm{N}$-(benzo[d][1,3]dioxol-5-yl(cyano)methyl)-4-methylbenzenesulfonamide (2i):

Sulfonylamidonitrile $\mathbf{2 i}$ was obtained $(30 \mathrm{mg}, 90 \%)$ as a pale-yellow oil. ${ }^{2}$ TLC: $R_{f} 0.35$ (2:1 heptanes/EtOAc). ${ }^{1} \mathrm{H}-\mathrm{NMR}\left(400 \mathrm{MHz}, \mathrm{CDCl}_{3}\right): \mathrm{d} 7.74(\mathrm{~d}, J=7.1 \mathrm{~Hz}, 2 \mathrm{H}), 7.43(\mathrm{~d}, J=7.1 \mathrm{~Hz}, 2 \mathrm{H})$, $7.21(\mathrm{~s}, 1 \mathrm{H}), 6.97(\mathrm{~d}, J=7.3 \mathrm{~Hz}, 1 \mathrm{H}), 6.73(\mathrm{~d}, J=7.3 \mathrm{~Hz}, 1 \mathrm{H}), 5.82(\mathrm{~s}, 2 \mathrm{H}), 5.34(\mathrm{~s}, 1 \mathrm{H}), 2.48(\mathrm{~s}$, 3H). ${ }^{13} \mathrm{C}-\mathrm{NMR}\left(100 \mathrm{MHz}, \mathrm{CDCl}_{3}\right): \mathrm{d} 149.2,146.3,142.5,141.0,128.7,128.3,126.5,121.4,118.6$, 110.8, 107.8, 101.6, 54.7, 22.6 ppm. ESI-MS m/z (rel int): (pos) 331.1 ([M+H] $\left.{ }^{+}, 100\right)$; (neg) 329.1 $\left(\left[\mathrm{M}-\mathrm{H}^{-}, 100\right)\right.$. 
<smiles>Cc1ccc(S(=O)(=O)NC(C#N)c2ccc(Cl)cc2)cc1</smiles>

N-((4-chlorophenyl)(cyano)methyl)-4-methylbenzenesulfonamide $\quad$ (2j): Purification by automated silica gel flash chromatography (10 g cartridge, $12 \mathrm{ml} / \mathrm{min}$. 20:1 heptanes/EtOAc to 1:4 heptanes/EtOAc over $12 \mathrm{~min}$ ) yielded sulfonylamidonitrile $\mathbf{2 j}$ ( $24 \mathrm{mg}, 75 \%)$ as a colorless oil. ${ }^{1}$ TLC: $R_{f} 0.69$ (2:1 heptanes/EtOAc). ${ }^{1} \mathrm{H}-\mathrm{NMR}\left(400 \mathrm{MHz}, \mathrm{CDCl}_{3}\right): \mathrm{d} 7.76(\mathrm{~d}, J=7.3 \mathrm{~Hz}, 2 \mathrm{H})$, 7.36-7.33 (m, 6H), 5.75 (bs, 1H), 5.45 (s, 1H), 2.48 (s, 3H). ${ }^{13}$ C-NMR (100 MHz, CDCl 3 ): d 140.8, 135.9, 135.7, 130.6, 130.0, 129.5, 128.4, 127.2, 116.0, 47.5, $21.6 \mathrm{ppm}$. ESI-MS $\mathrm{m} / \mathrm{z}$ (rel int): (pos) $321.1\left([\mathrm{M}+\mathrm{H}]^{+}, 100\right)$; (neg) $319.1\left([\mathrm{M}-\mathrm{H}]^{-}, 100\right)$.

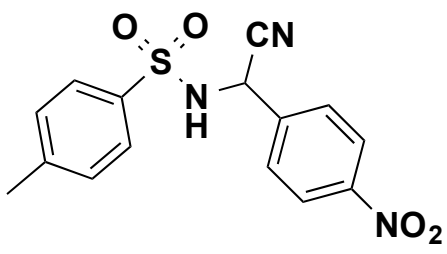

$\mathbf{N}$-(cyano(4-nitrophenyl)methyl)-4-methylbenzenesulfonamide (2k): Purification by automated silica gel flash chromatography (10 g cartridge, $12 \mathrm{ml} / \mathrm{min}$. 20:1 heptanes/EtOAc to 1:4 heptanes/EtOAc over $12 \mathrm{~min})$ yielded sulfonylamidonitrile $\mathbf{2 k}(26 \mathrm{mg}, 80 \%)$ as a white solid. ${ }^{1}$ TLC: $R_{f} 0.58$ (2:1 heptanes/EtOAc). ${ }^{1} \mathrm{H}-\mathrm{NMR}\left(400 \mathrm{MHz}, \mathrm{CDCl}_{3}\right): \mathrm{d} 8.24(\mathrm{~d}, J=7.0 \mathrm{~Hz}, 2 \mathrm{H}), 7.78$ (d, $J=7.0 \mathrm{~Hz}, 2 \mathrm{H}), 7.19(\mathrm{~d}, J=7.0 \mathrm{~Hz}, 2 \mathrm{H}), 7.49(\mathrm{~d}, J=7.0 \mathrm{~Hz}, 2 \mathrm{H}), 5.34(\mathrm{~s}, 1 \mathrm{H}), 2.48(\mathrm{~s}, 3 \mathrm{H}) .{ }^{13} \mathrm{C}-\mathrm{NMR}$ $\left(100 \mathrm{MHz}_{1} \mathrm{CDCl}_{3}\right): \mathrm{d} 148.5,142.1,141.2,138.4,129.6,128.1,118.0,54.1,22.6$ ppm. ESI-MS m/z (rel int): (pos) $332.2\left([\mathrm{M}+\mathrm{H}]^{+}, 100\right)$; (neg) $330.2\left([\mathrm{M}-\mathrm{H}]^{-}, 100\right)$. 


\section{SYNTHESIS OF SULFONYLAMIDONITRILES FROM TABLE 3:}<smiles>CS(=O)(=O)NC(C#N)CCc1ccccc1</smiles>

$\mathbf{N}$-(1-cyano-3-phenylpropyl)methanesulfonamide (2I): Purification by automated silica gel flash chromatography (10 g cartridge, $12 \mathrm{ml} / \mathrm{min}$. 20:1 heptanes/EtOAc to 1:4 heptanes/EtOAc over $12 \mathrm{~min}$ ) yielded indole $\mathbf{2 l}(22 \mathrm{mg}, 92 \%)$ as a colorless oil. TLC: $R_{f} 0.51$ (2:1 heptanes/EtOAc). ${ }^{1} \mathrm{H}-\mathrm{NMR}\left(400 \mathrm{MHz}, \mathrm{CDCl}_{3}\right): \mathrm{d}$ 7.28-7.20 (m, 5H), 5.61 (bs, $\left.1 \mathrm{H}\right), 4.26(\mathrm{t}, \mathrm{J}=7.1 \mathrm{~Hz}, 1 \mathrm{H}), 3.05(\mathrm{~s}$, $3 \mathrm{H}), 2.79(\mathrm{t}, J=6.6 \mathrm{~Hz}, 2 \mathrm{H}), 2.17(\mathrm{tt}, J=7.1,6.6 \mathrm{~Hz}, 2 \mathrm{H}) .{ }^{13} \mathrm{C}-\mathrm{NMR}\left(100 \mathrm{MHz}, \mathrm{CDCl}_{3}\right): \mathrm{d} 138.6$, $128.8,128.4,126.8,118.2,43.8,41.3,35.0,31.1$ ppm. ESI-MS $m / z$ (rel int): (pos) $239.1\left([\mathrm{M}+\mathrm{H}]^{+}\right.$, 100); (neg) $237.1\left([\mathrm{M}-\mathrm{H}]^{-}, 100\right)$.<smiles>N#CC(CCc1ccccc1)NS(=O)(=O)c1cccs1</smiles>

$\mathbf{N}$-(1-cyano-3-phenylpropyl)thiophene-2-sulfonamide $(2 \mathrm{~m})$ : Sulfonylamidonitrile $\mathbf{2 m}$ was obtained (27 mg, 90\%) as a pale-yellow oil. TLC: $R_{f} 0.40$ (2:1 heptanes/EtOAc). ${ }^{1} \mathrm{H}-\mathrm{NMR}$ $\left(400 \mathrm{MHz}, \mathrm{CDCl}_{3}\right): \mathrm{d} 7.65(\mathrm{~d}, J=7.3 \mathrm{~Hz}, 2 \mathrm{H}), 7.28-7.16(\mathrm{~m}, 6 \mathrm{H}), 4.26(\mathrm{t}, J=6.5 \mathrm{~Hz}, 1 \mathrm{H}), 2.75(\mathrm{t}, J=$ $6.2 \mathrm{~Hz}, 2 \mathrm{H}), 2.15(\mathrm{tt}, J=6.6,6.2 \mathrm{~Hz}, 2 \mathrm{H}) .{ }^{13} \mathrm{C}-\mathrm{NMR}\left(100 \mathrm{MHz}, \mathrm{CDCl}_{3}\right): \mathrm{d}$ 138.6, 133.6, 133.4, 128.9, 128.4, 127.8, 126.8, 117.0, 44.0, 35.4, $31.1 \mathrm{ppm}$. ESI-MS $\mathrm{m} / \mathrm{z}$ (rel int): (pos) 307.2 $\left([\mathrm{M}+\mathrm{H}]^{+}, 100\right) ;$ (neg) $305.2\left([\mathrm{M}-\mathrm{H}]^{-}, 100\right)$.<smiles>N#CC(CCc1ccccc1)NS(=O)(=O)c1ccc([N+](=O)[O-])cc1</smiles>

$\mathbf{N}$-(1-cyano-3-phenylpropyl)-4-nitrobenzenesulfonamide (2n): Sulfonylamidonitrile $\mathbf{2 n}$ was obtained (33 mg, 97\%) as a white solid. TLC: $R_{f} 0.57$ (2:1 heptanes/EtOAc). ${ }^{1} \mathrm{H}-\mathrm{NMR}(400 \mathrm{MHz}$, $\left.\mathrm{CDCl}_{3}\right): \mathrm{d} 8.35(\mathrm{~d}, J=7.1 \mathrm{~Hz}, 2 \mathrm{H}), 8.04(\mathrm{~d}, J=7.1 \mathrm{~Hz}, 2 \mathrm{H}), 7.28-7.16(\mathrm{~m}, 5 \mathrm{H}), 4.22(\mathrm{t}, J=6.4 \mathrm{~Hz}$, $1 \mathrm{H}), 2.79(\mathrm{t}, J=6.6 \mathrm{~Hz}, 2 \mathrm{H}), 2.20(\mathrm{tt}, J=6.9,6.4 \mathrm{~Hz}, 2 \mathrm{H}), 1.27$ (bs, $1 \mathrm{H}) .{ }^{13} \mathrm{C}-\mathrm{NMR}(100 \mathrm{MHz}$, $\left.\mathrm{CDCl}_{3}\right): \mathrm{d}$ 150.4, 144.5, 138.3, 128.9, 128.8, 128.5, 128.4, 128.3, 127.0, 124.6, 116.9, 43.8, 35.1, 31.0 ppm. ESI-MS m/z (rel int): (pos) 346.2 ([M+H] $\left.]^{+}, 100\right)$; (neg) 344.2 ([M-H] $\left.{ }^{-}, 100\right)$. 
<smiles>N#CC(CCc1ccccc1)NS(=O)(=O)C(F)(F)F</smiles>

$\mathbf{N}$-(1-cyano-3-phenylpropyl)trifluoromethanesulfonamide (20): Sulfonylamidonitrile 20 was obtained (26 mg, 91\%) as a pale-yellow oil. TLC: $R_{f} 0.58$ (2:1 heptanes/EtOAc). ${ }^{1} \mathbf{H}-\mathbf{N M R}$ $\left(400 \mathrm{MHz} \mathrm{CDCl}_{3}\right): \mathrm{d} 7.32-7.27(\mathrm{~m}, 5 \mathrm{H}), 4.42(\mathrm{t}, J=6.8 \mathrm{~Hz}, 1 \mathrm{H}), 2.77(\mathrm{t}, J=6.6 \mathrm{~Hz}, 2 \mathrm{H}), 2.24(\mathrm{tt}, J=$ 6.9, $6.5 \mathrm{~Hz}, 2 \mathrm{H}) .{ }^{13} \mathrm{C}-\mathrm{NMR}\left(100 \mathrm{MHz}, \mathrm{CDCl}_{3}\right): \mathrm{d} 138.0,128.9,128.4,127.1,126.6,120.8(\mathrm{q}, \mathrm{J}=$ $250 \mathrm{~Hz}, \mathrm{CF}_{3}$ ), 116.6, 45.0, 35.6, 31.0 ppm. ESI-MS m/z (rel int): (pos) 293.1 ([M+H] $\left.]^{+}, 100\right)$; (neg) $291.1\left([\mathrm{M}-\mathrm{H}]^{-}, 100\right)$.<smiles>COc1ccc(C(C#N)NS(=O)(=O)c2ccc([N+](=O)[O-])cc2)cc1</smiles>

$\mathrm{N}$-(cyano(4-methoxyphenyl)methyl)-4-nitrobenzenesulfonamide $\quad(2 q)$ : Purification by automated silica gel flash chromatography (10 g cartridge, $12 \mathrm{ml} / \mathrm{min}$. 20:1 heptanes/EtOAc to 1:4 heptanes/EtOAc over $12 \mathrm{~min}$ ) yielded sulfonylamidonitrile $\mathbf{2 q}(30 \mathrm{mg}, 86 \%)$ as a white solid. TLC: $R_{f} 0.40$ (2:1 heptanes/EtOAc). ${ }^{1} \mathrm{H}-\mathrm{NMR}\left(400 \mathrm{MHz}, \mathrm{CDCl}_{3}\right): \mathrm{d} 8.42(\mathrm{~d}, J=7.3 \mathrm{~Hz}, 2 \mathrm{H}), 8.08$ (d, $J=7.3 \mathrm{~Hz}, 2 \mathrm{H}), 7.28(\mathrm{~d}, J=7.1 \mathrm{~Hz}, 2 \mathrm{H}), 6.88(\mathrm{~d}, J=7.1 \mathrm{~Hz}, 2 \mathrm{H}), 5.52(\mathrm{~s}, 1 \mathrm{H}), 3.78(\mathrm{~s}, 3 \mathrm{H}) .{ }^{13} \mathrm{C}-\mathrm{NMR}$ $\left(100 \mathrm{MHz}, \mathrm{CDCl}_{3}\right): \mathrm{d}$ 160.9, 150.2, 144.9, 128.7, 128.6, 128.3, 124.6, 123.1, 116.0, 114.5, 55.4, 47.9 ppm. ESI-MS m/z (rel int): (pos) 348.2 ([M+H $\left.]^{+}, 100\right)$; (neg) $346.2\left([\mathrm{M}-\mathrm{H}]^{-}, 100\right)$.

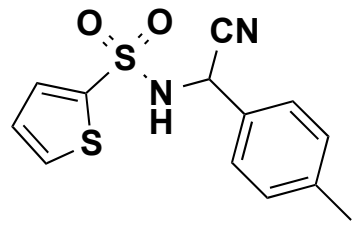

$\boldsymbol{N}$-(cyano(p-tolyl)methyl)thiophene-2-sulfonamide (2r): Sulfonylamidonitrile $\mathbf{2 r}$ was obtained (26 mg, 90\%) as a pale-yellow oil. TLC: $R_{f} 0.45$ (2:1 heptanes/EtOAc). ${ }^{1} \mathrm{H}-\mathbf{N M R}\left(400 \mathrm{MHz}, \mathrm{CDCl}_{3}\right)$ : d $7.70(\mathrm{~d}, J=8.8 \mathrm{~Hz}, 2 \mathrm{H}), 7.29(\mathrm{~d}, J=7.1 \mathrm{~Hz}, 2 \mathrm{H}), 7.21(\mathrm{~d}, J=7.1 \mathrm{~Hz}, 2 \mathrm{H}), 7.16(\mathrm{~d}, J=8.8 \mathrm{~Hz}, 1 \mathrm{H})$, $5.50(\mathrm{~s}, 1 \mathrm{H}), 5.41(\mathrm{~s}, 1 \mathrm{H}), 2.35(\mathrm{~s}, 3 \mathrm{H}) .{ }^{13} \mathrm{C}-\mathrm{NMR}\left(100 \mathrm{MHz}, \mathrm{CDCl}_{3}\right): \mathrm{d}$ 140.1, 139.8, 133.6, 133.4, 130.0, 128.7, 127.8, 127.0, 116.2, 48.1, 21.1 ppm. ESI-MS m/z (rel int): (pos) $293.1\left([\mathrm{M}+\mathrm{H}]^{+}\right.$, 100); (neg) $291.1\left([\mathrm{M}-\mathrm{H}]^{-}, 100\right)$.

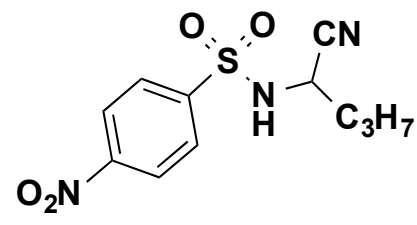

$\mathbf{N}$-(1-cyanobutyl)-4-nitrobenzenesulfonamide (2s): Sulfonylamidonitrile $2 \mathrm{~s}$ was obtained (26 mg, 93\%) as a pale-yellow oil. TLC: $R_{f} 0.62$ (2:1 heptanes/EtOAc). ${ }^{1} \mathbf{H}-\mathbf{N M R}\left(400 \mathrm{MHz}, \mathrm{CDCl}_{3}\right)$ : d $8.42(\mathrm{~d}, J=7.1 \mathrm{~Hz}, 2 \mathrm{H}), 8.08(\mathrm{~d}, J=7.1 \mathrm{~Hz}, 2 \mathrm{H}), 4.26(\mathrm{t}, J=6.2 \mathrm{~Hz}, 1 \mathrm{H}), 1.76(\mathrm{dt}, J=7.3,6.9 \mathrm{~Hz}$, $2 \mathrm{H}), 1.54-1.50(\mathrm{~m}, 2 \mathrm{H}), 0.99(\mathrm{t}, J=6.8 \mathrm{~Hz}, 3 \mathrm{H}) .{ }^{13} \mathrm{C}-\mathrm{NMR}\left(100 \mathrm{MHz}, \mathrm{CDCl}_{3}\right): \mathrm{d} 150.4,144.8$, 
130.6, 128.6, 124.6, 117.2, 44.3, 35.6, 18.4, 13.0 ppm. ESI-MS m/z (rel int): (pos) $284.1\left([\mathrm{M}+\mathrm{H}]^{+}\right.$, $100) ;$ (neg) $282.1\left([\mathrm{M}-\mathrm{H}]^{-}, 100\right)$.<smiles>CCCCC(C#N)NS(=O)(=O)c1ccc([N+](=O)[O-])cc1</smiles>

$\mathbf{N}$-(1-cyanooctyl)-4-nitrobenzenesulfonamide (2t): Sulfonylamidonitrile $2 \mathrm{t}$ was obtained (31 mg, 90\%) as a pale-yellow oil. TLC: $R_{f} 0.66$ (2:1 heptanes/EtOAc). ${ }^{1} \mathbf{H}-\mathbf{N M R}\left(400 \mathrm{MHz}, \mathrm{CDCl}_{3}\right)$ : d $8.48(\mathrm{~d}, J=7.3 \mathrm{~Hz}, 2 \mathrm{H}), 8.12(\mathrm{~d}, J=7.1 \mathrm{~Hz}, 2 \mathrm{H}), 4.26(\mathrm{t}, J=6.4 \mathrm{~Hz}, 1 \mathrm{H}), 1.80(\mathrm{dt}, J=7.3,6.9 \mathrm{~Hz}$, $2 \mathrm{H}), 1.52-1.48(\mathrm{~m}, 2 \mathrm{H}), 1.32-1.25(\mathrm{~m}, 8 \mathrm{H}), 0.84(\mathrm{t}, J=6.8 \mathrm{~Hz}, 3 \mathrm{H}) .{ }^{13} \mathrm{C}-\mathrm{NMR}\left(100 \mathrm{MHz}, \mathrm{CDCl}_{3}\right)$ : d $150.4,144.8,128.5,124.6,117.2,44.5,33.7,31.5,28.8,28.8,25.0,22.5,14.0$ ppm. ESI-MS $\mathrm{m} / \mathrm{z}$ (rel int): (pos) $340.1\left([\mathrm{M}+\mathrm{H}]^{+}, 100\right)$; (neg) $338.1\left([\mathrm{M}-\mathrm{H}]^{-}, 100\right)$. 
PROTON

NVC-2015-II-17-1P
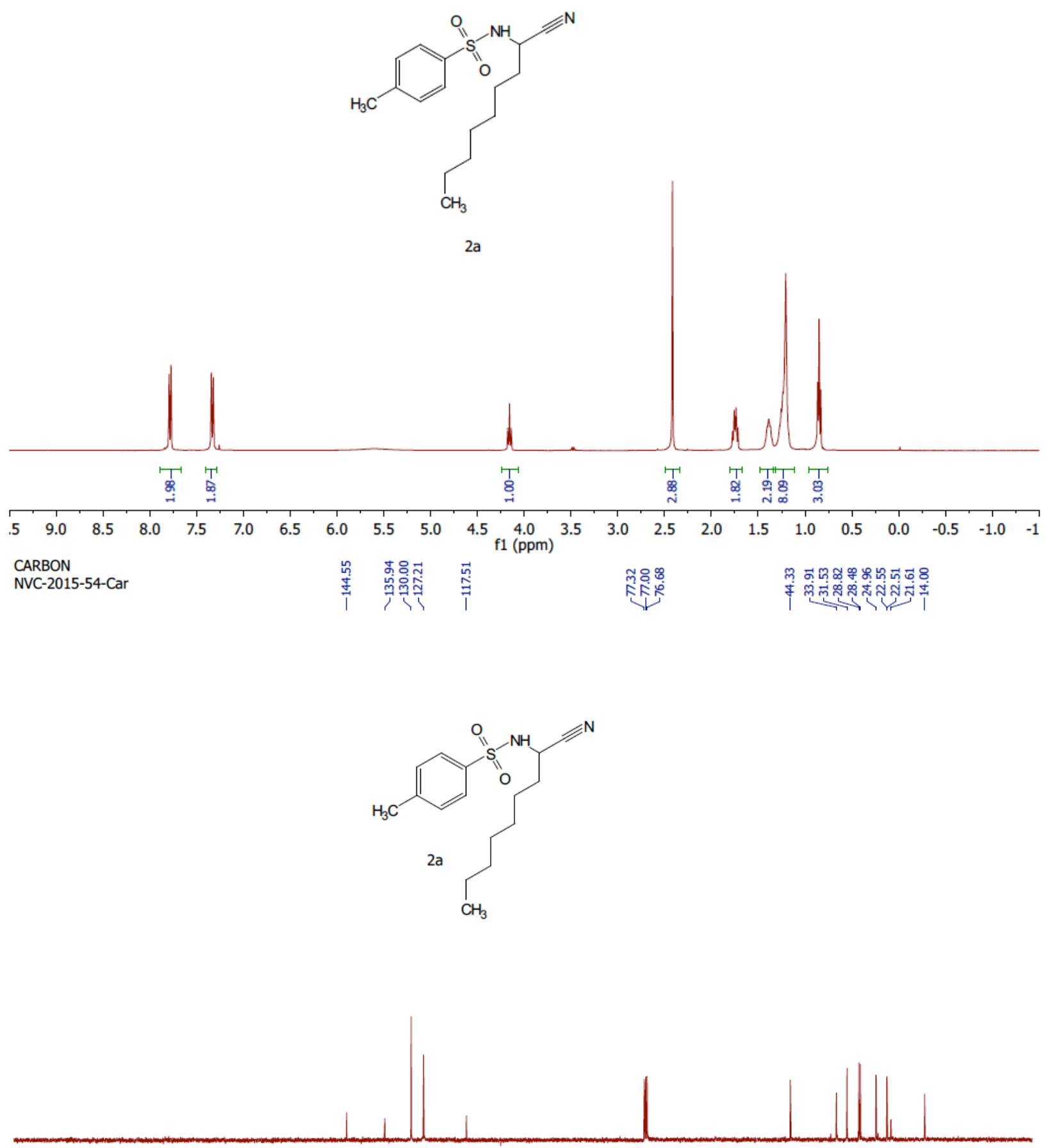

$\begin{array}{lllllllllllllllllllllll}210 & 200 & 190 & 180 & 170 & 160 & 150 & 140 & 130 & 120 & \begin{array}{c}110 \\ \mathrm{f} 1(\mathrm{ppm})\end{array} & 90 & 80 & 70 & 60 & 50 & 40 & 30 & 20 & 10 & 0 & -1\end{array}$ 
PROTON

NVC-2015-II-33-1P
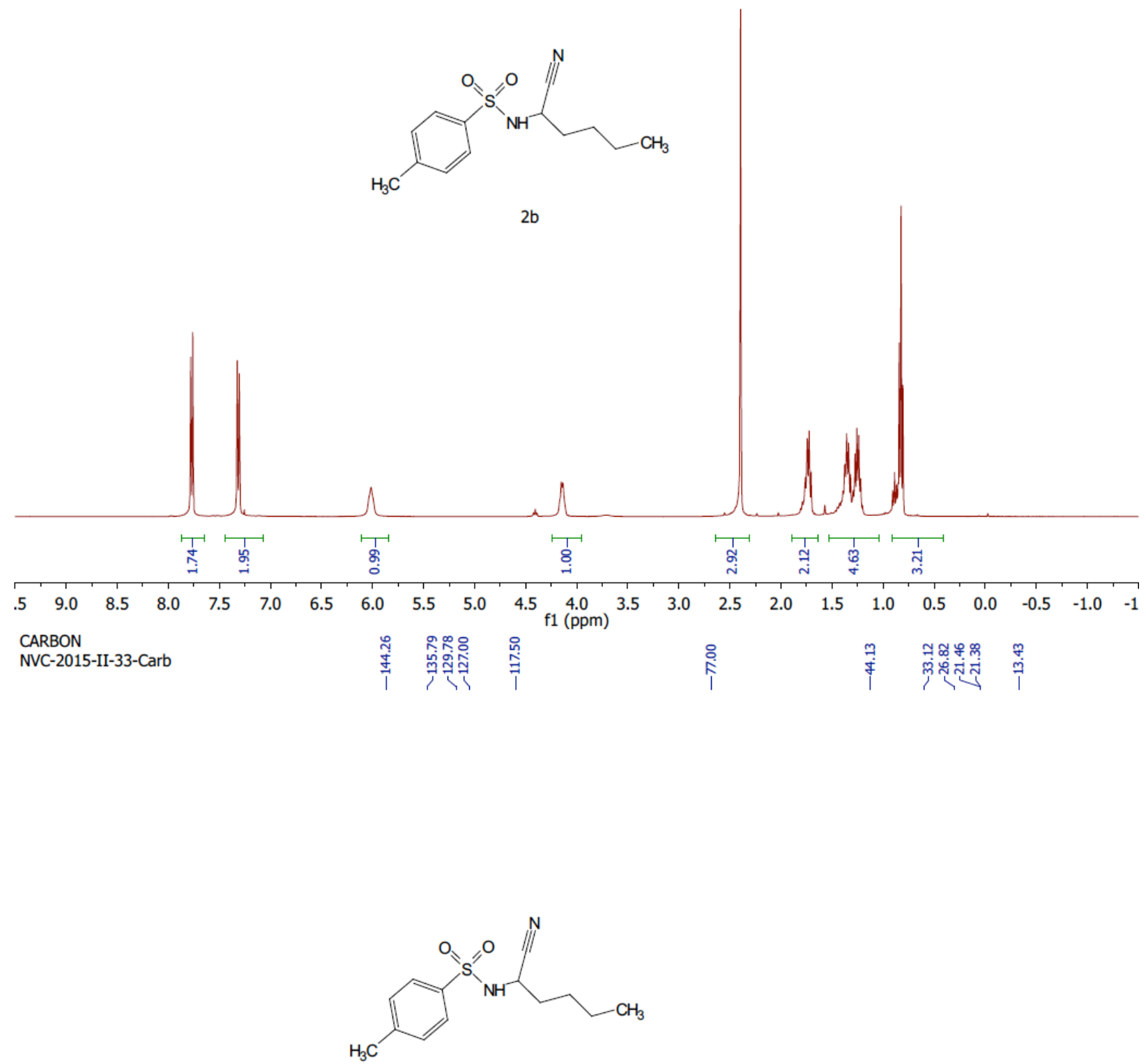

$2 b$

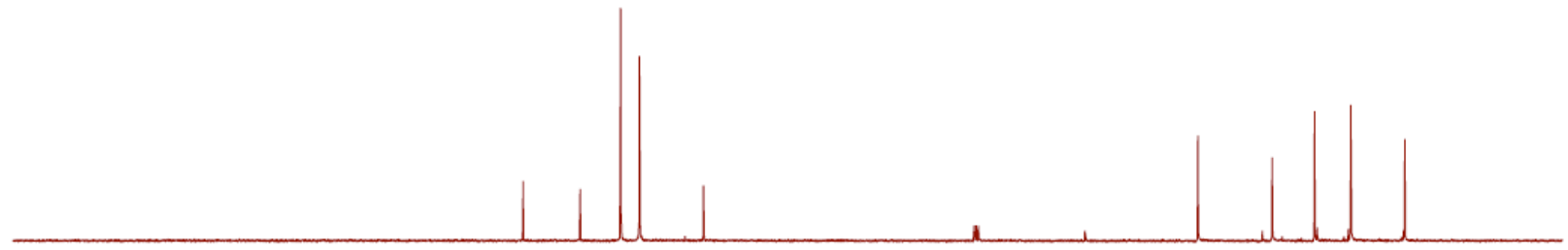

$20 \quad 210 \quad 200 \quad 190$

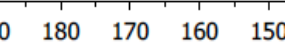

150140

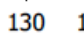

$\begin{array}{lll}120 & 110 & 100 \\ \mathrm{f} 1(\mathrm{ppm})\end{array}$

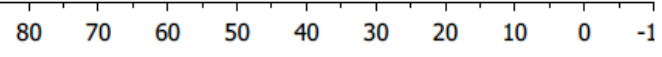


PROTON

ADB-2015-II-6-P

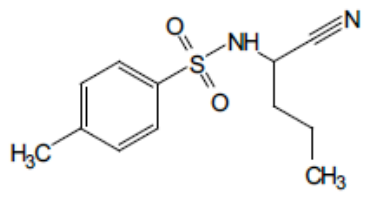

$2 \mathrm{c}$
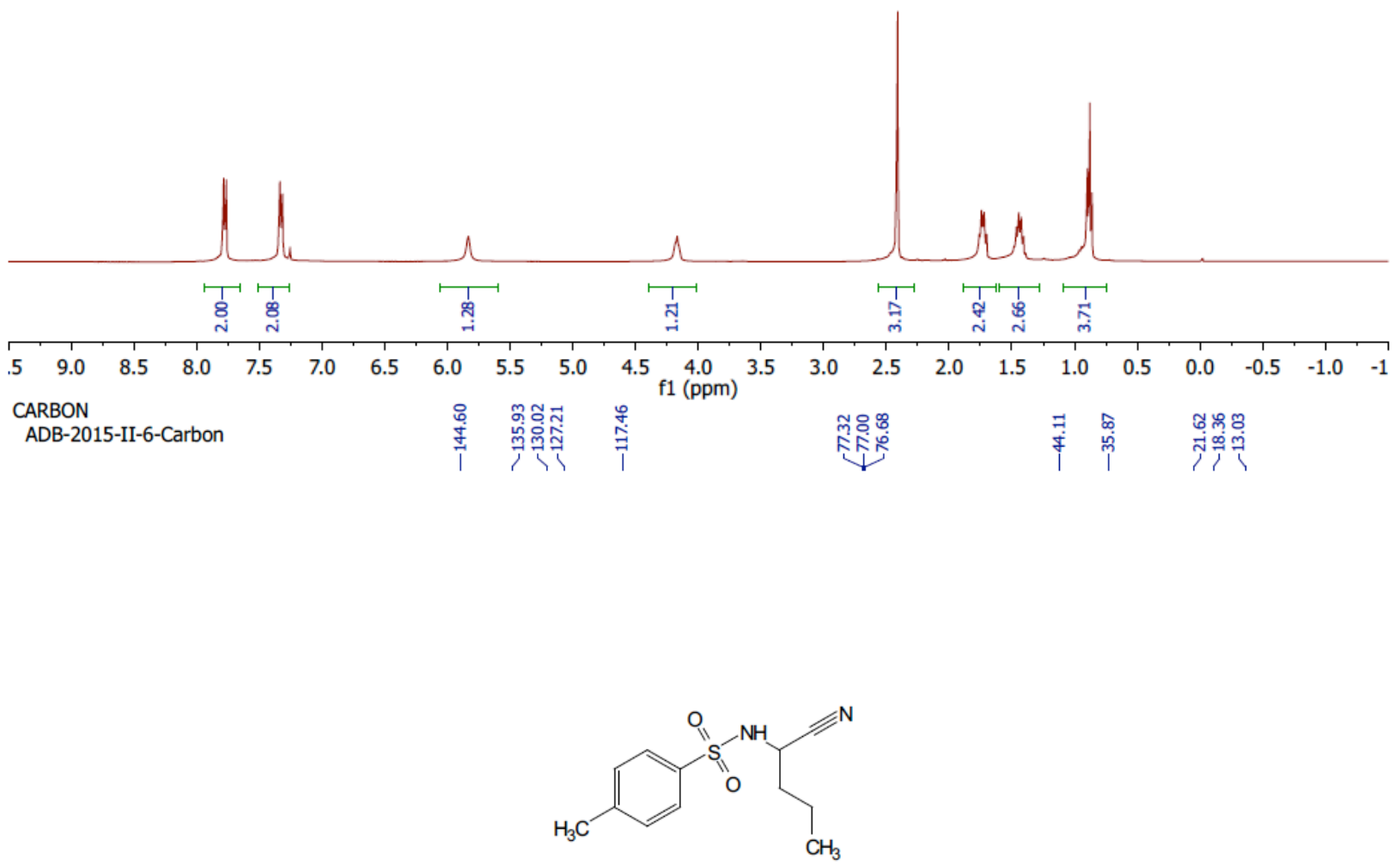

$2 c$

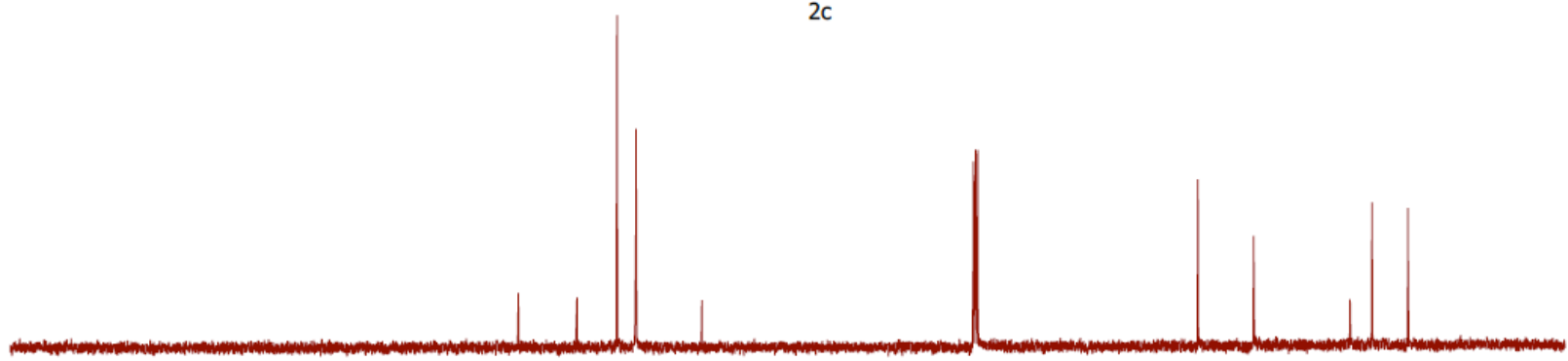

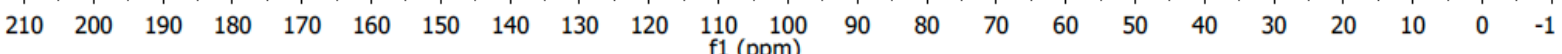


PROTON

GH-2015-98-1P
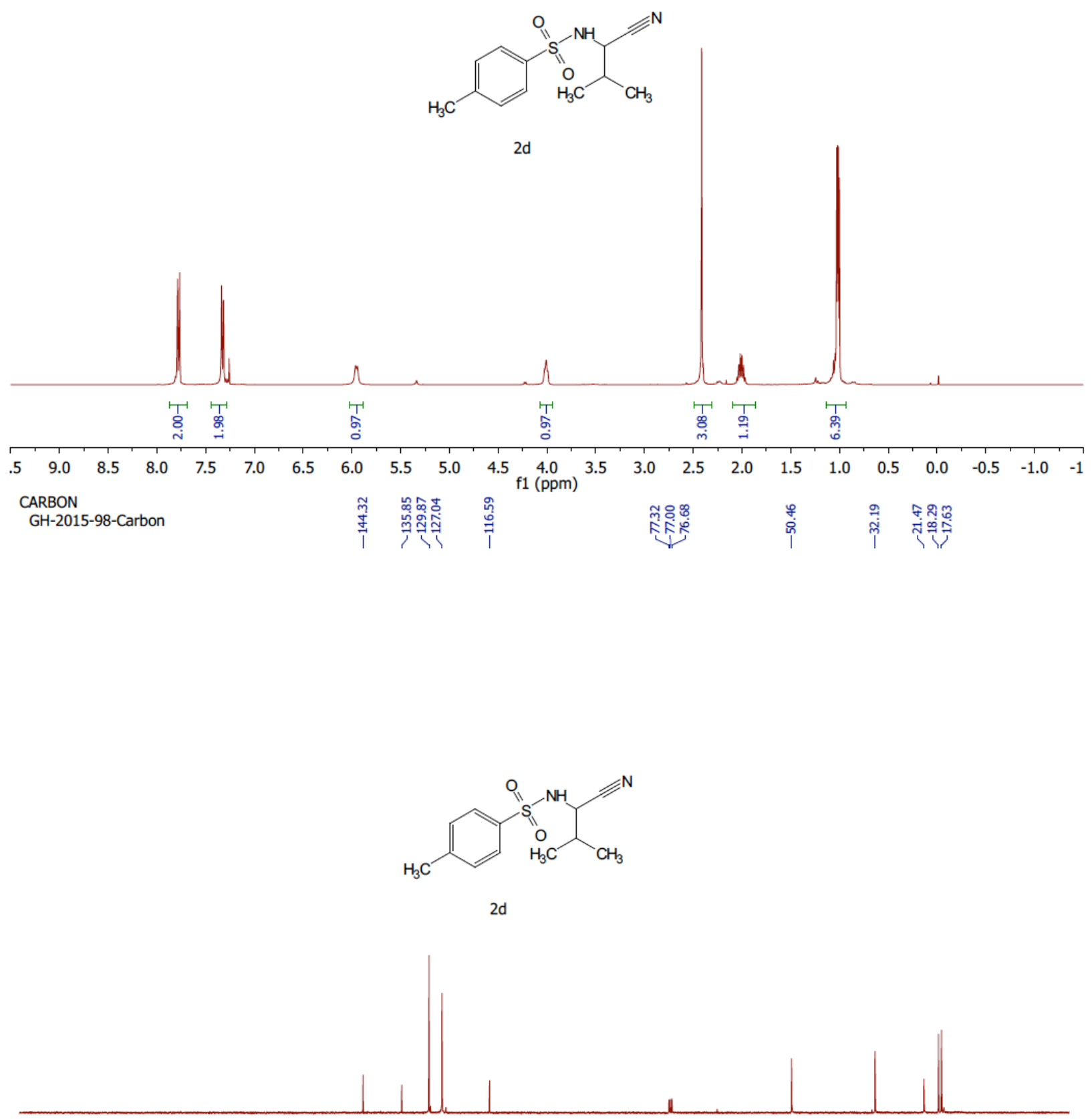

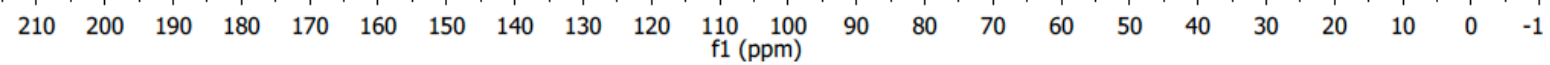


PROTON

GH-2015-64-P2
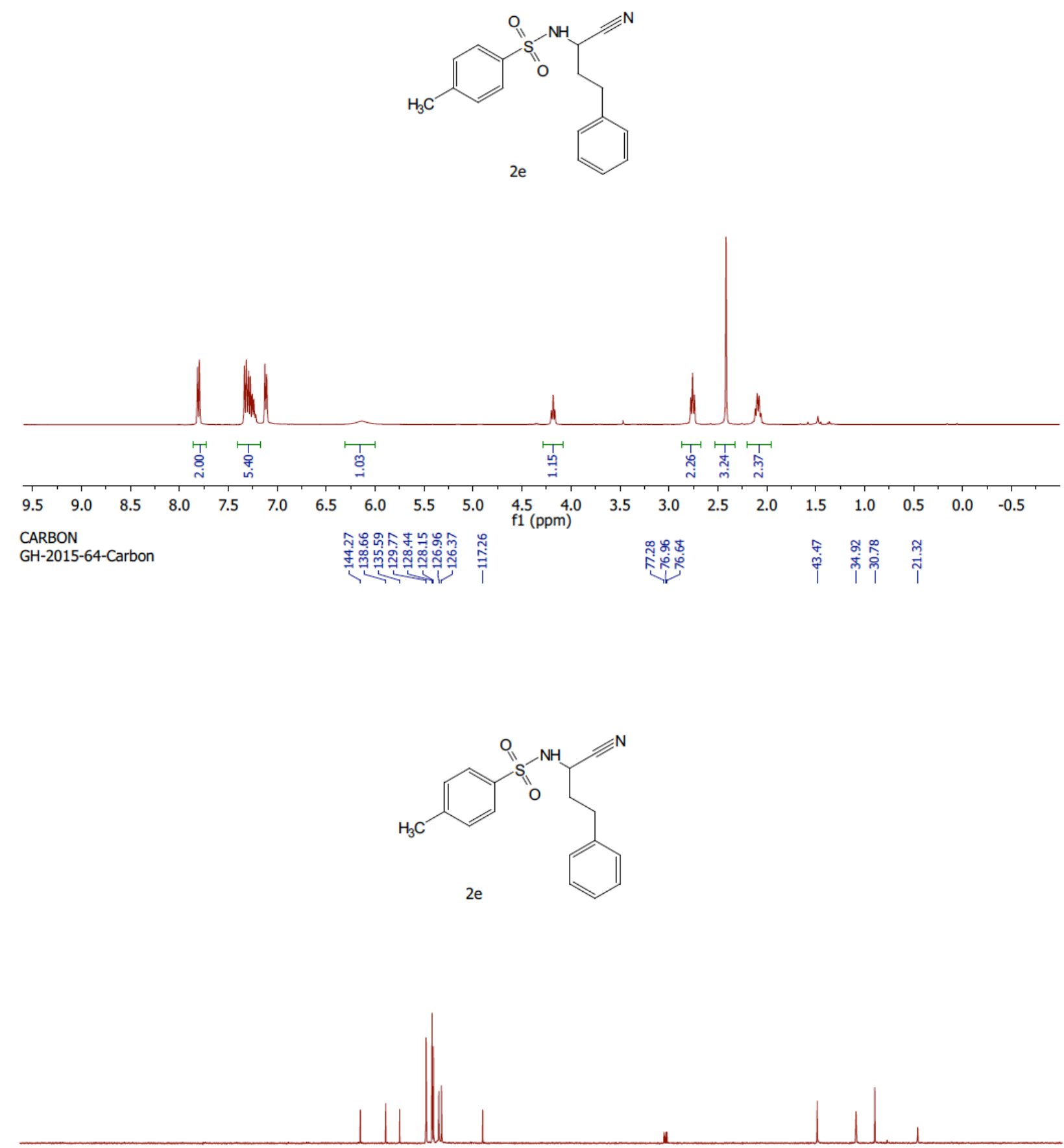

$\begin{array}{llllllllllllllllllllllll}210 & 200 & 190 & 180 & 170 & 160 & 150 & 140 & 130 & 120 & \begin{array}{c}110 \\ \mathrm{f} 1(\mathrm{ppm})\end{array} & 90 & 80 & 70 & 60 & 50 & 40 & 30 & 20 & 10 & 0 & -1\end{array}$


PROTON

ADB-2015-137-2P
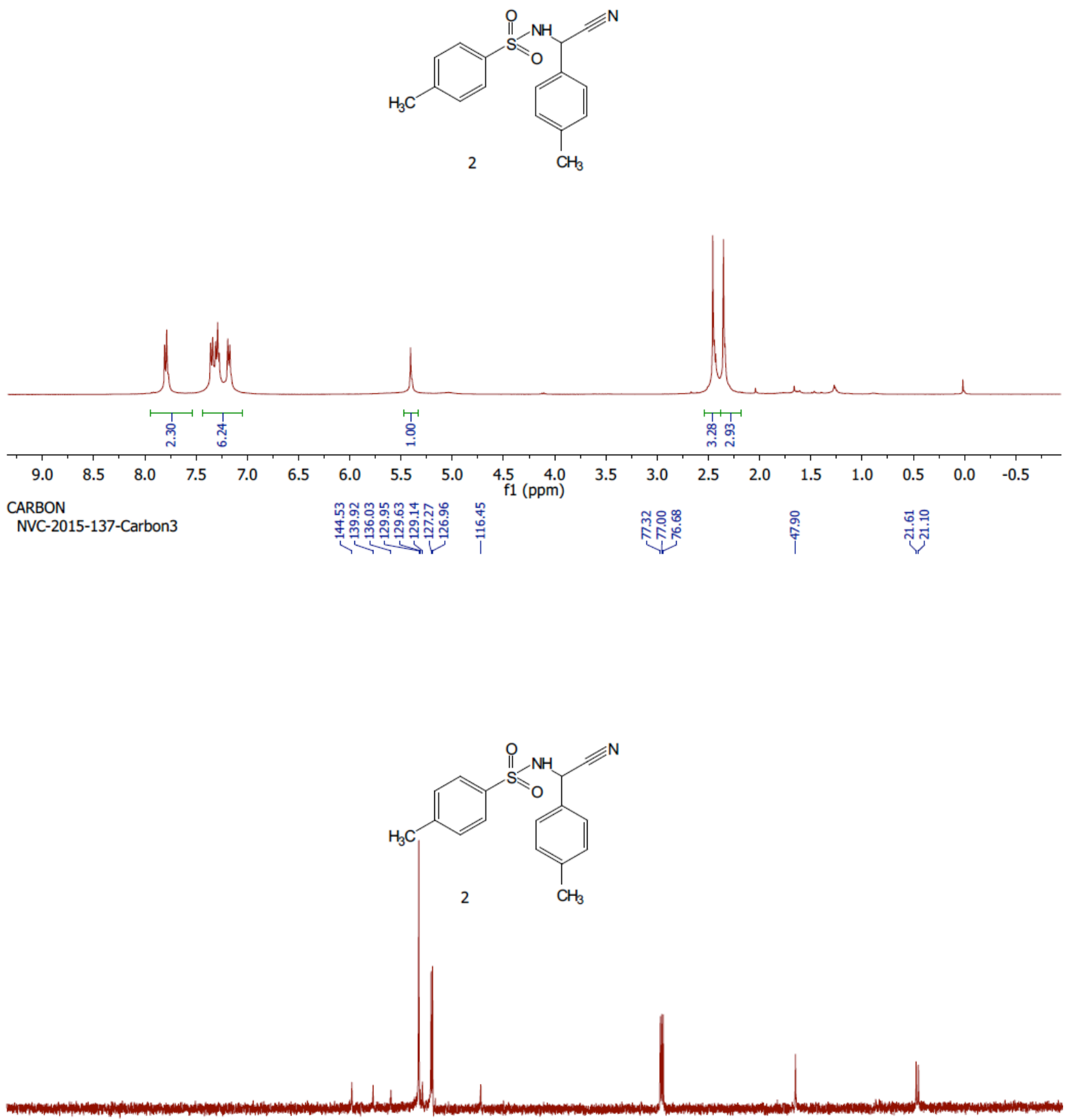

\begin{tabular}{|c|c|c|c|c|c|c|c|c|c|c|c|c|c|c|c|c|c|c|}
\hline 210 & 200 & 190 & 180 & 17 & 160 & 150 & 140 & 130 & 120 & $110 \quad 100$ & 90 & 80 & 70 & 60 & 50 & 40 & & -1 \\
\hline
\end{tabular}


PROTON

NVC-2015-67-2P
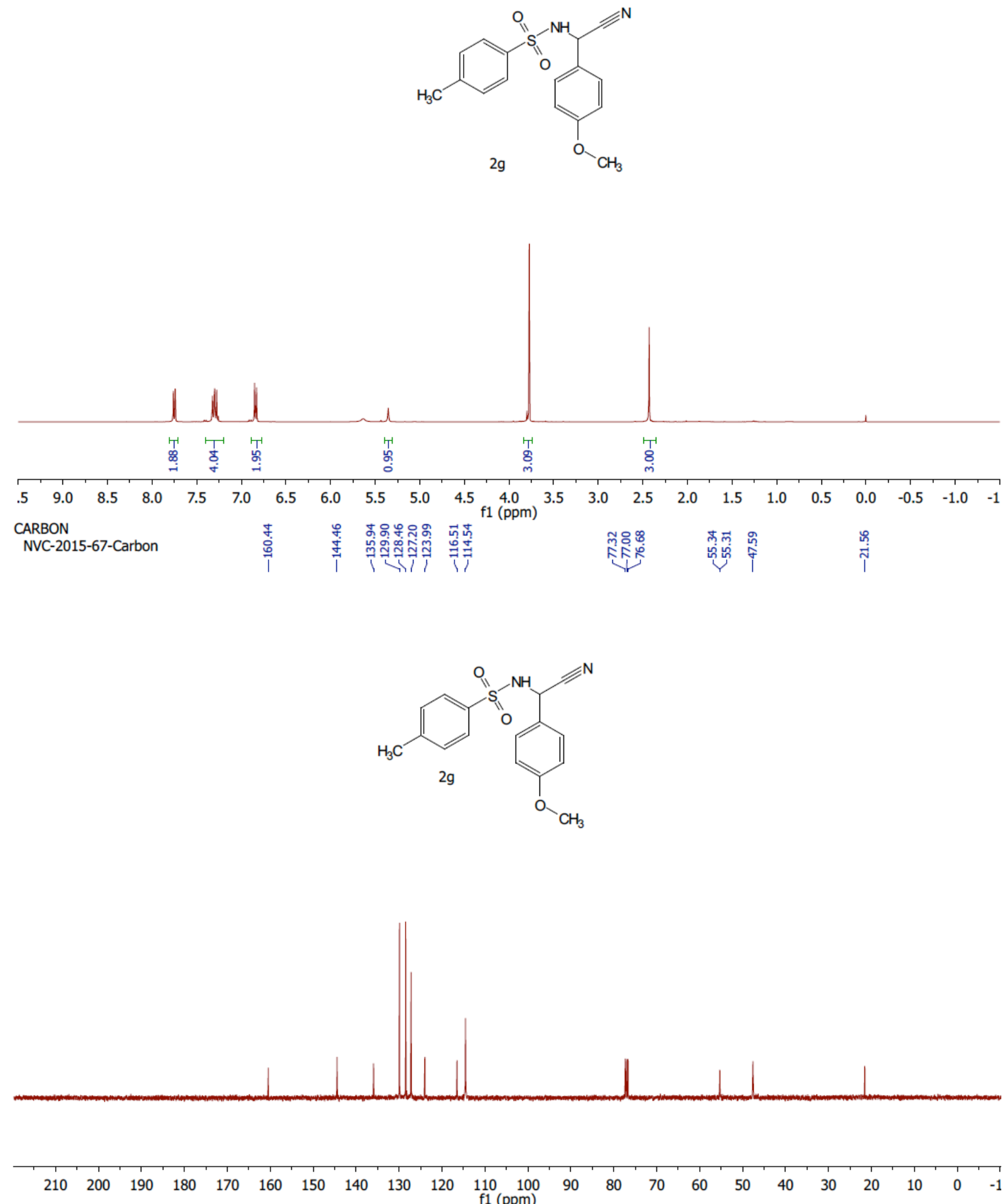
PROTON

ADB-2015-II-1-Pure

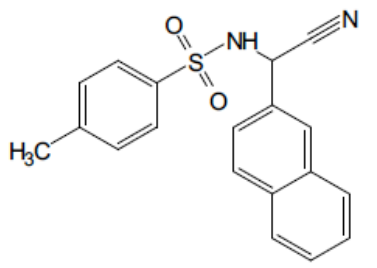

$2 \mathrm{~h}$
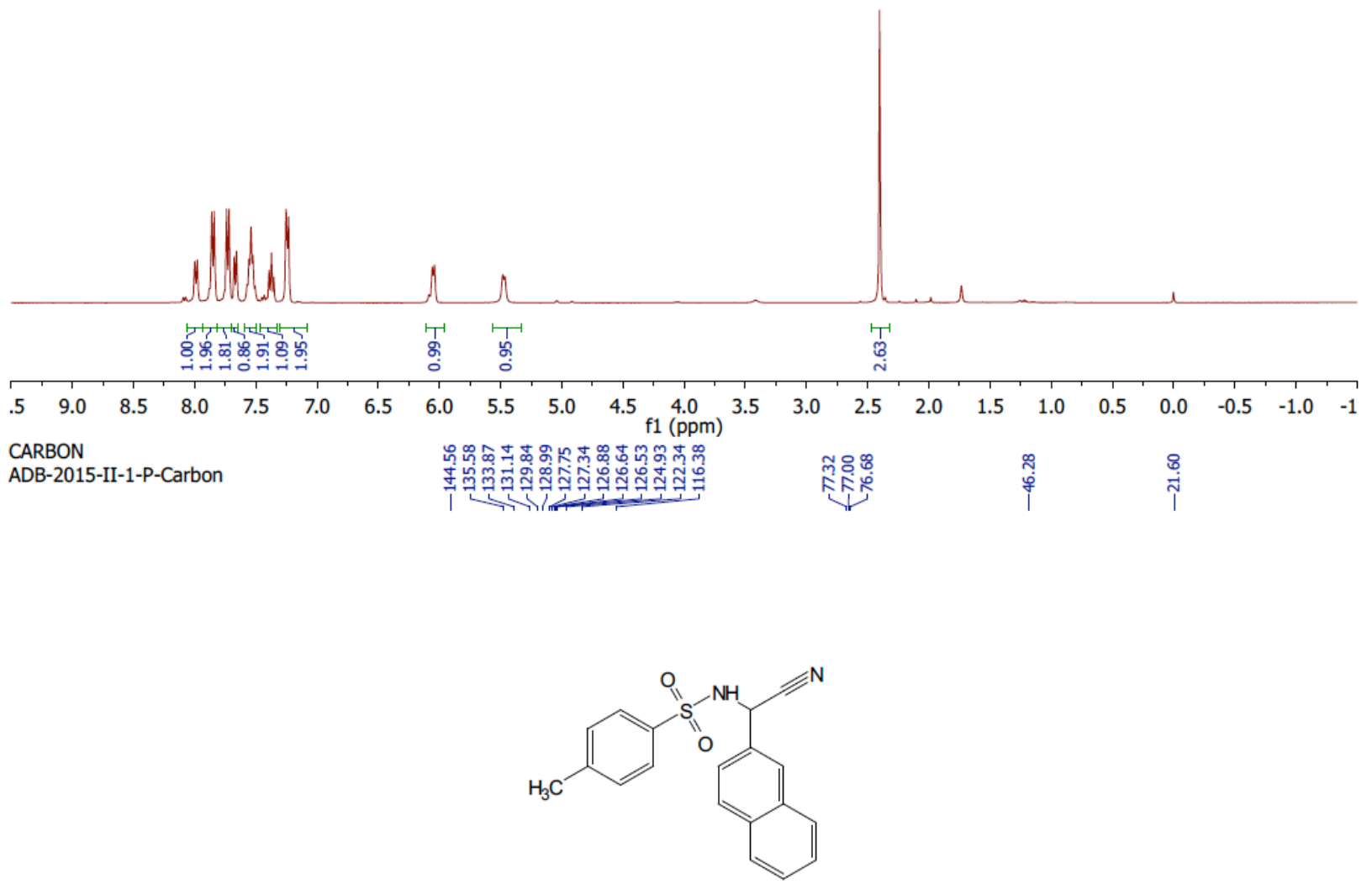

$2 \mathrm{~h}$

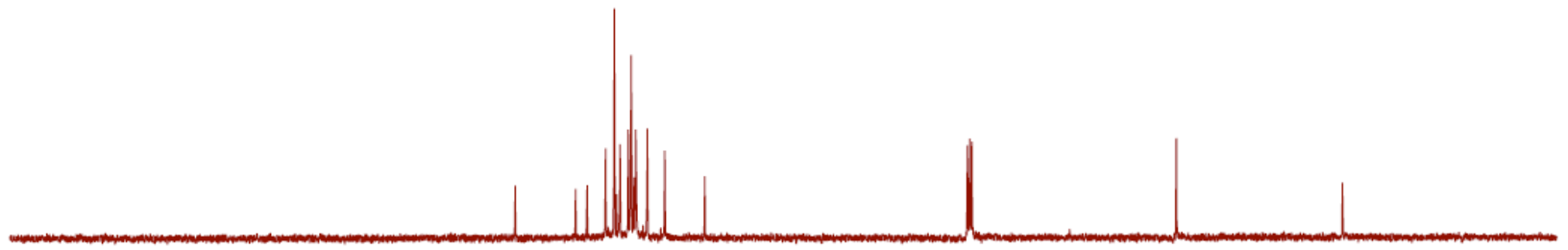

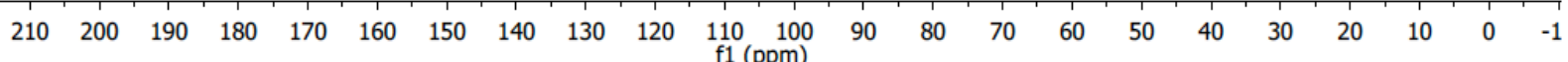


PROTON

ADB-2015-II-2real-1P
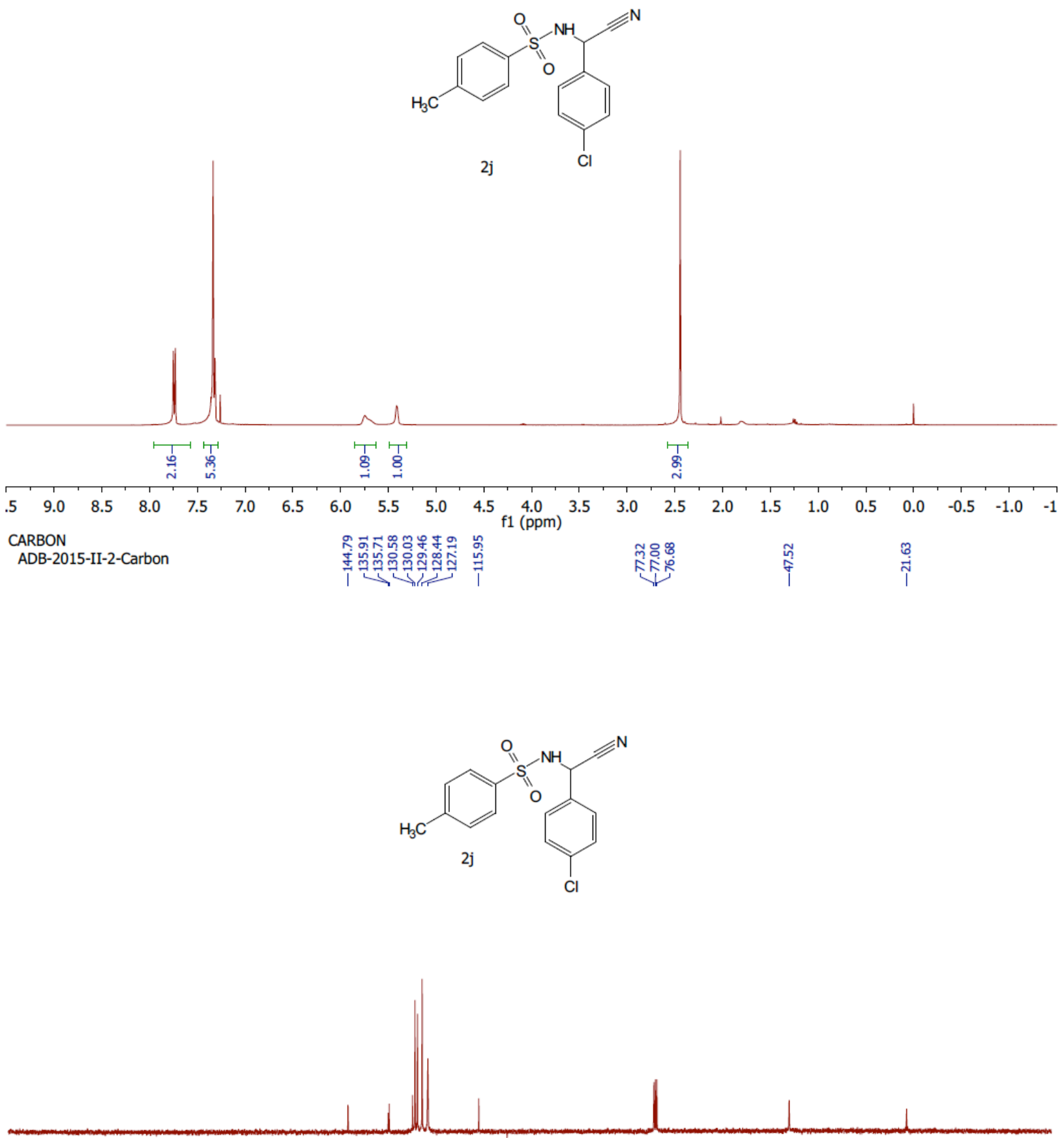

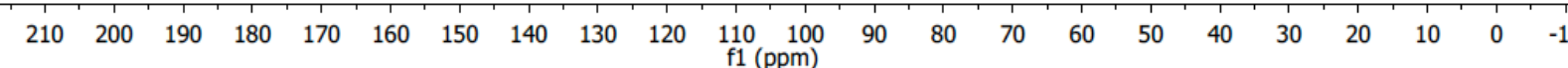


PROTON

NVC-2015-97-2P
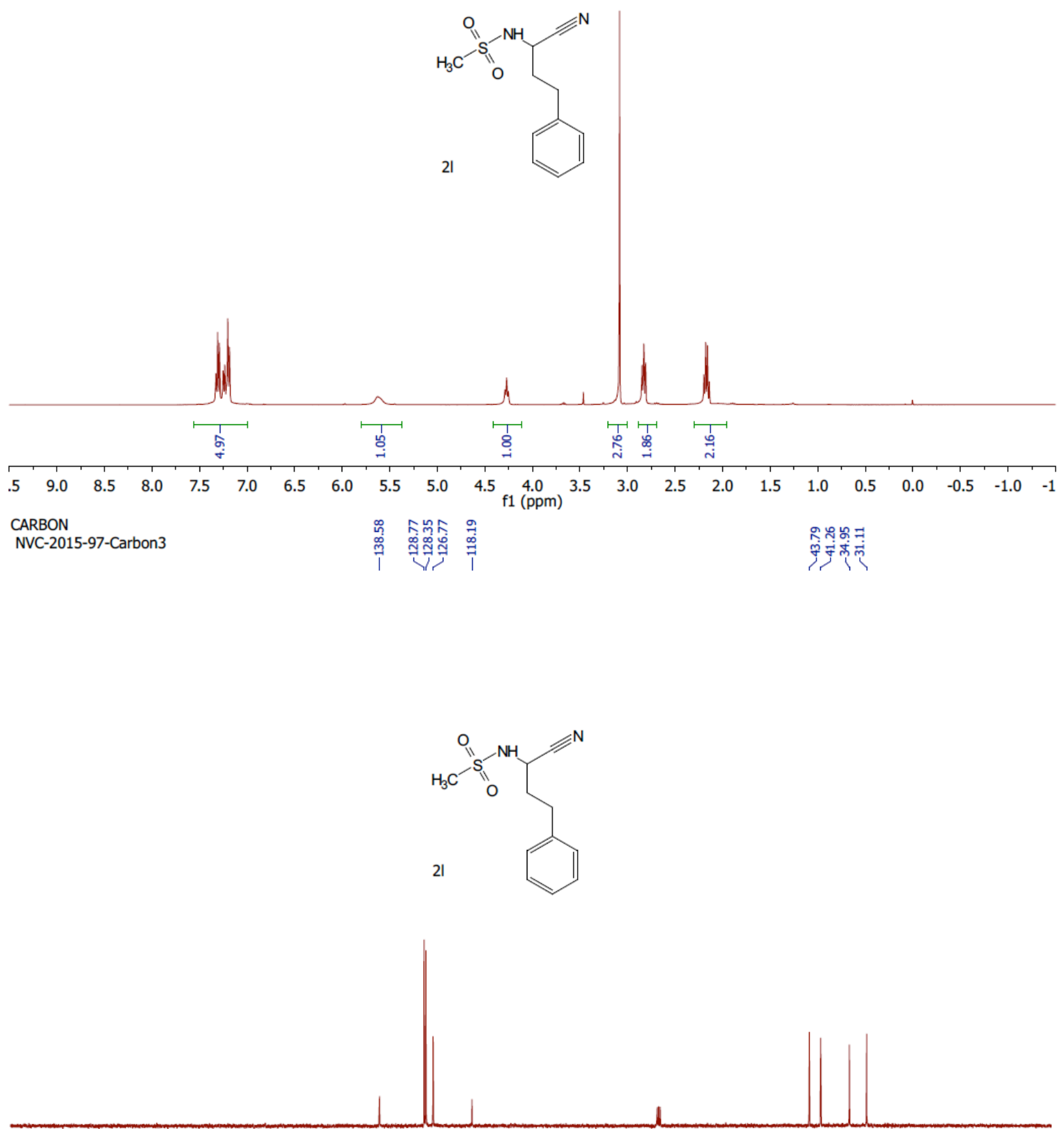

$\begin{array}{llllllllllllllllllllllllllllll}20 & 210 & 200 & 190 & 180 & 170 & 160 & 150 & 140 & 130 & 120 & 110 & 100 & 90 & 80 & 70 & 60 & 50 & 40 & 30 & 20 & 10 & 0 & -1\end{array}$ 
PROTON

NVC-2015-96-2P
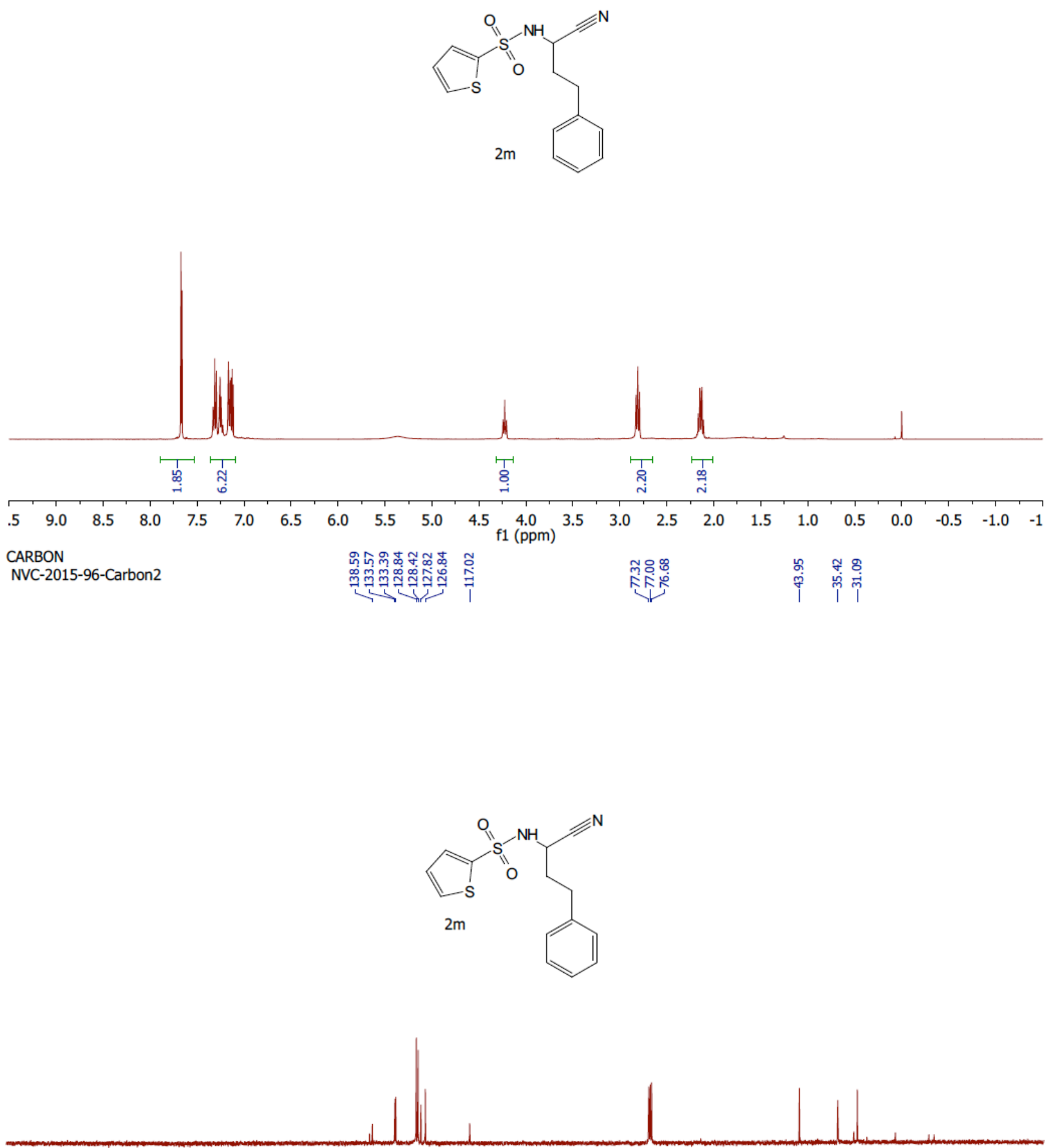

$\begin{array}{llllllllllllllllllllllll}210 & 200 & 190 & 180 & 170 & 160 & 150 & 140 & 130 & 120 & \underset{f 10}{110}\left(\begin{array}{ll}100 \\ \mathrm{fmm})\end{array}\right. & 90 & 80 & 70 & 60 & 50 & 40 & 30 & 20 & 10 & 0 & -1\end{array}$ 
PROTON

GH-2015-71B-1P

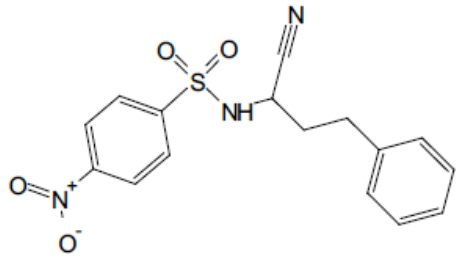

$2 n$

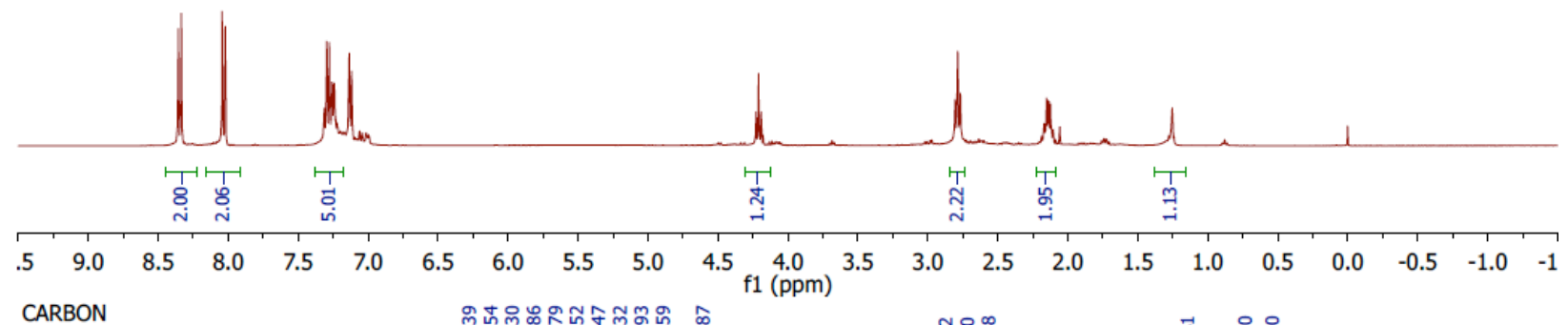
CARBON

GH-2015-71B-Carbon

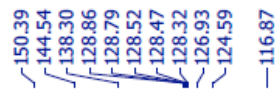

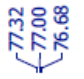

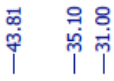

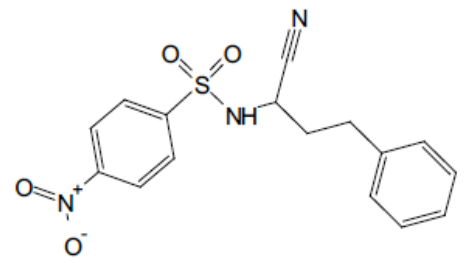

$2 n$

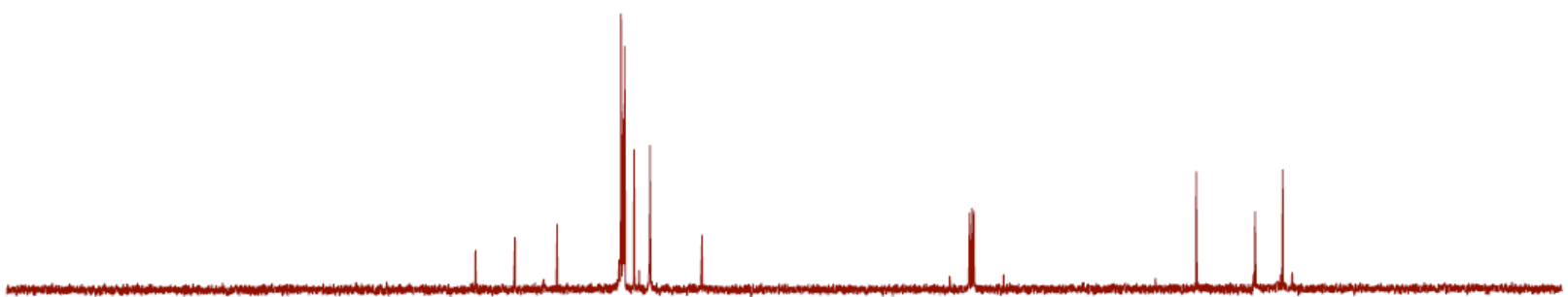

$\begin{array}{llllllllllll}210 & 200 & 190 & 180 & 170 & 160 & 150 & 140 & 130 & 120 & \begin{array}{l}110 \\ \mathrm{f} 1(\mathrm{ppm})\end{array}\end{array}$

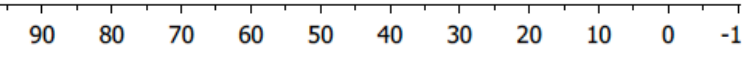


PROTON

GH-2015-100-1P

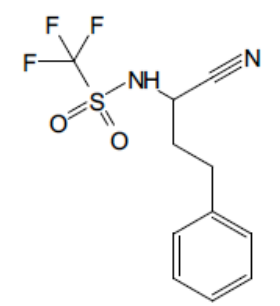

20
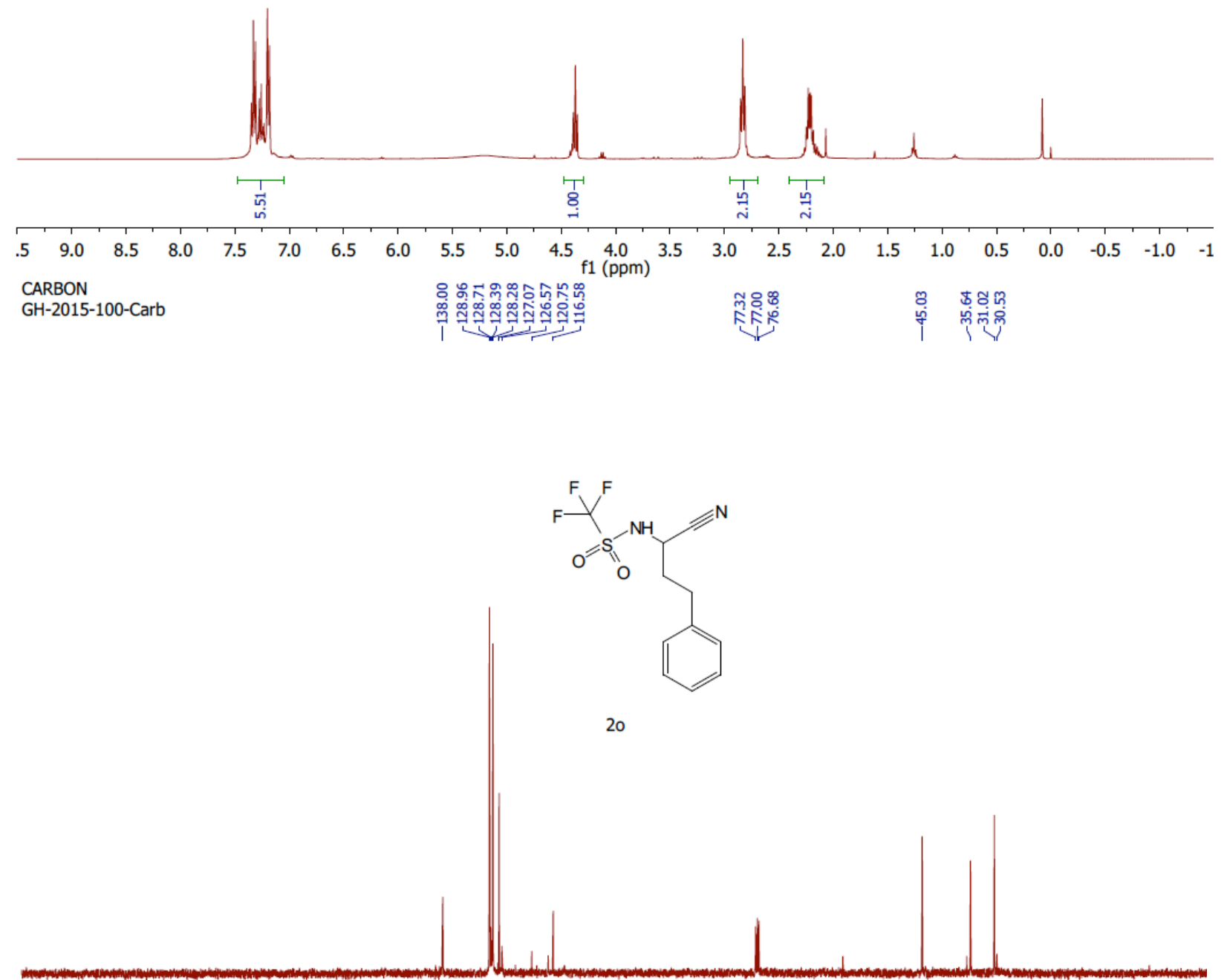

$\begin{array}{lllllllllllllllllllllll}210 & 200 & 190 & 180 & 170 & 160 & 150 & 140 & 130 & 120 & \begin{array}{c}110 \\ \mathrm{f} 1(\mathrm{ppm})\end{array} & 100 & 80 & 70 & 60 & 50 & 40 & 30 & 20 & 10 & 0 & -1\end{array}$


PROTON

NVC-2015-111-2P
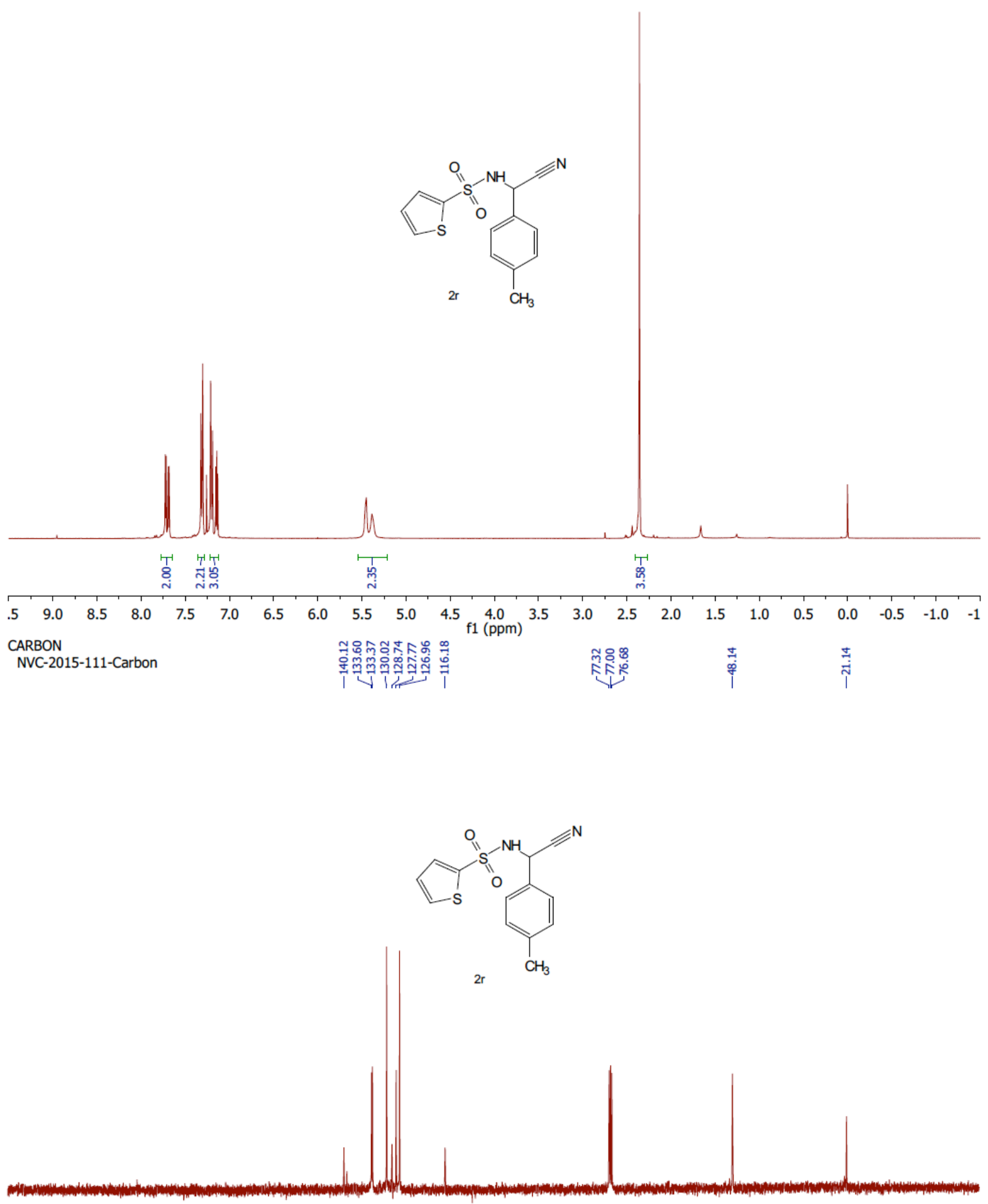

$\begin{array}{lllllllllllllllllllllll}210 & 200 & 190 & 180 & 170 & 160 & 150 & 140 & 130 & 120 & \begin{array}{c}110 \\ \mathrm{f} 1(\mathrm{ppm})\end{array} & 90 & 80 & 70 & 60 & 50 & 40 & 30 & 20 & 10 & 0 & -1\end{array}$


PROTON

NVC-2015-90-3P

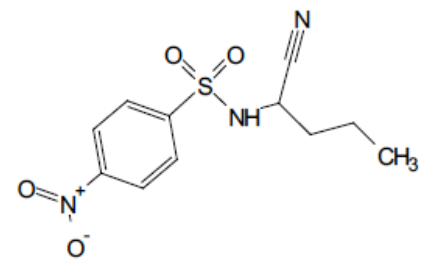

$2 s$
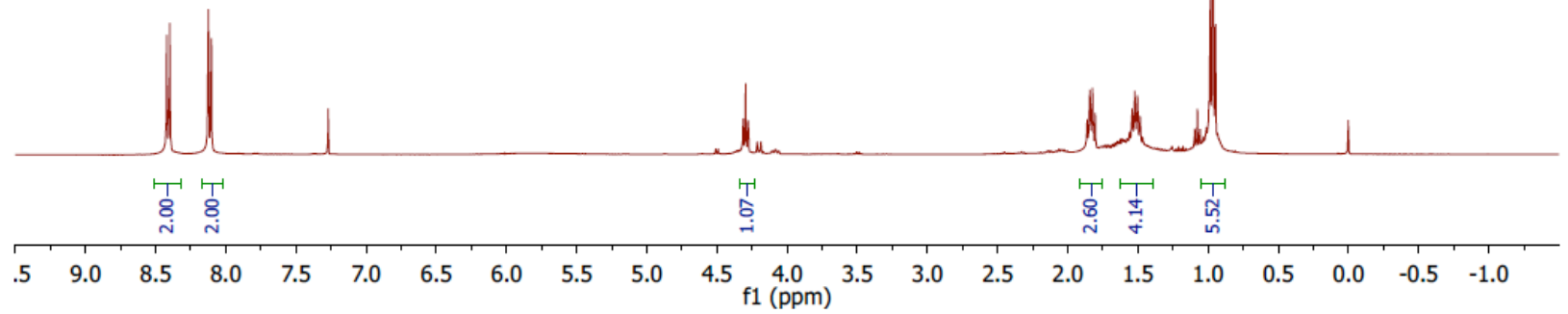

CARBON

NVC-2015-90-Carbon2

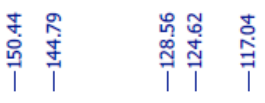

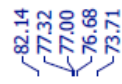

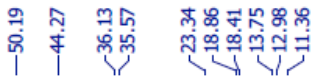

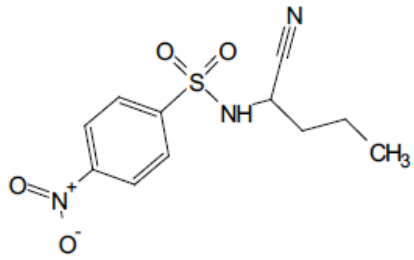

2s

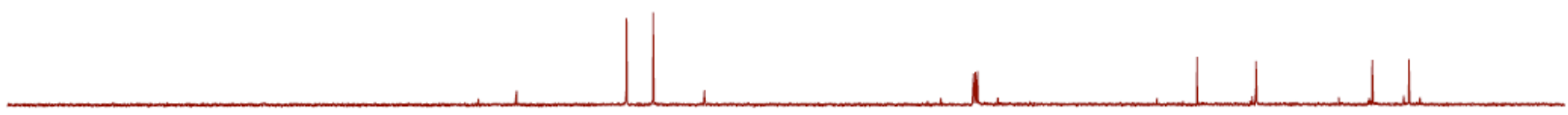

\begin{tabular}{|c|c|c|c|c|c|c|c|c|c|c|c|c|c|c|c|c|c|c|c|c|c|c|}
\hline 210 & 200 & 190 & 180 & 170 & 160 & 150 & 140 & 130 & 120 & 110 & $\begin{array}{c}100 \\
\mathrm{pm})\end{array}$ & 90 & 80 & 70 & 60 & 50 & 40 & 30 & 20 & 10 & 0 & -1 \\
\hline
\end{tabular}


PROTON

ADB-2015-145-P2

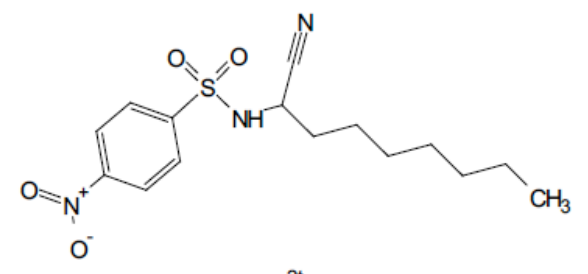

$2 \mathrm{t}$
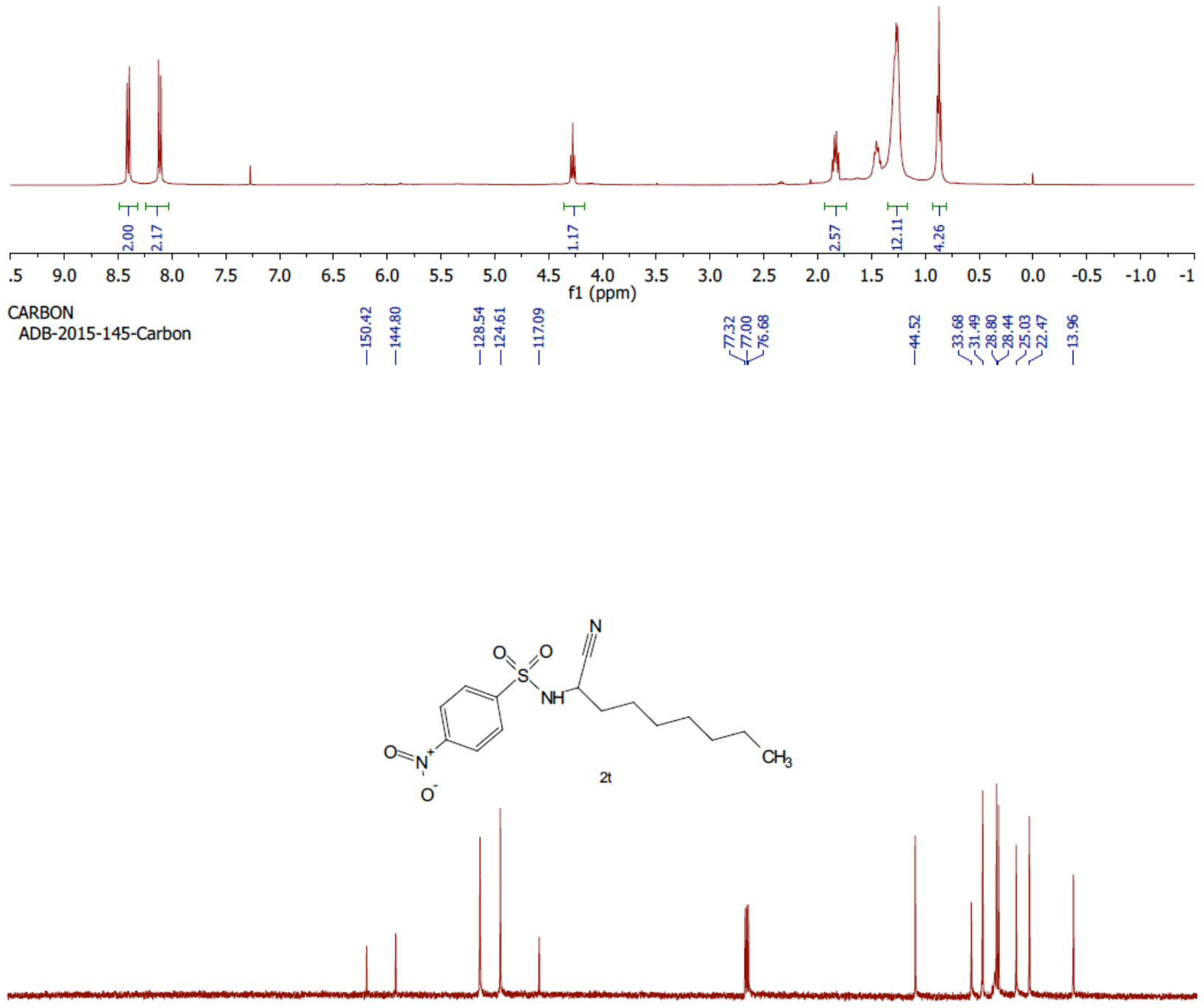

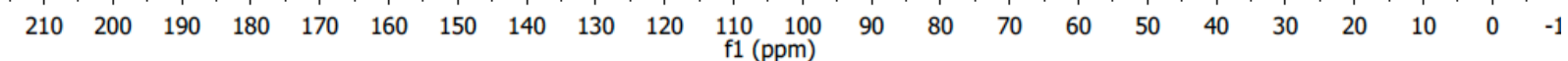




\section{F. REFERENCES:}

1. Das, B.; Balasubramanyan, P.; Krishnaiah, M.; Veeranjaneyulu, B.; Reddy, G. C. Synthesis 2009, 20, 3467-3471.

2. Wang, J.; Hu, X.; Jiang, J.; Gou, S.; Huang, X.; Liu, X.; Feng, X. Angew. Chem. Int. Ed. 2007, $46,8468-8470$.

3. Kano, T.; Sasaki, K.; Konishi, T.; Mii, H.; Maruoka, K. Tetrahedron Lett. 2006, 47, 46164618.

4. He, H.-X.; Du, D.-M. Eur. J. Org. Chem. 2014, 6190-6199.

5. Wang, W.; Wang, Y.; Wu, B.; Cong, R.; Gao, W.; Qin, B.; Yang, T. Cat. Commun. 2015, 174178. 